\title{
Nickel-Catalyzed Double Dehydrogenative Coupling of Secondary Alcohols and $\beta$-Amino Alcohols to Access Substituted Pyrroles
}

\author{
Anitha Alanthadka, ${ }^{\ddagger}$ Sourajit Bera, ${ }^{\ddagger}$ Mari Vellakkaran and Debasis Banerjee* \\ (A. A. ${ }^{\ddagger}$ and S. B. ${ }^{\ddagger}$ contributed equally) \\ Department of Chemistry \\ Laboratory of Catalysis and Organic Synthesis \\ Indian Institute of Technology Roorkee, Roorkee-247667, Uttarakhand, India \\ E-mail: dbane.fcy@iitr.ac.in
}

\section{Table of Contents}

Nickel catalyzed synthesis of pyrroles

S2

${ }^{1} \mathrm{H}$ NMR and ${ }^{13} \mathrm{C}$ NMR for selected compounds

S10 


\section{[1.1] Nickel-catalysed synthesis of pyrroles:}

Table S1: Screening of Nickel catalysts ${ }^{a}$

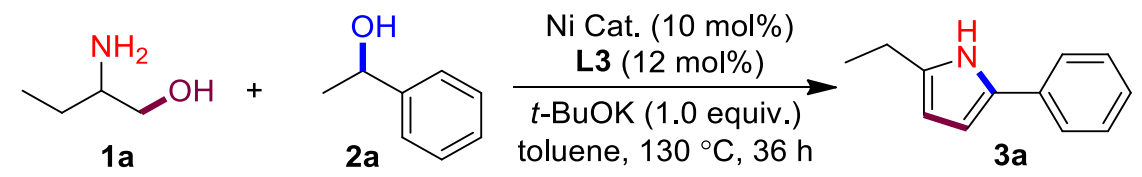

\begin{tabular}{|c|c|c|c|}
\hline Entry & Ni-catalyst & Base & GC-MS Conversion 3a \\
\hline 1. & $\mathrm{NiCl}_{2}$ & $t$-BuOK & 17 \\
\hline $\mathbf{2 .}$ & NiBr2 & $t$-BuOK & $\mathbf{2 8}$ \\
\hline 3. & $\mathrm{Ni}(\mathrm{acac})_{2}$ & $t$-BuOK & 18 \\
\hline 4. & $\mathrm{NiCl}_{2}$.DME & $t$-BuOK & 7 \\
\hline 5. & $\mathrm{Ni}(\mathrm{COD})_{2}$ & $t$-BuOK & 8 \\
\hline
\end{tabular}

Reaction condition: ${ }^{2}$ 2-aminobutan-1-ol 1a $(0.25 \mathrm{mmol}), 2 \mathrm{a}(0.5 \mathrm{mmol}), \mathrm{Ni}$-catalyst $(10 \mathrm{~mol} \%), \mathbf{L 3}(12 \mathrm{~mol} \%), t$ BuOK (1.0 equiv.), toluene $(1.0 \mathrm{~mL})$, Schenk tube under $\mathrm{N}_{2}$ atmosphere, $130{ }^{\circ} \mathrm{C}$ in oil bath, $36 \mathrm{~h}$ reaction time.

Table S2: Screening of base ${ }^{a}$

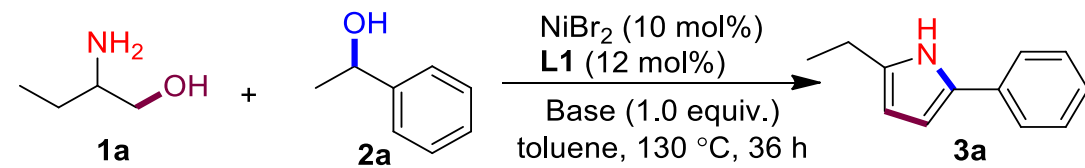

\begin{tabular}{|c|c|c|}
\hline Entry & Base & GC-MS Conversion 3a \\
\hline 1. & $t$-BuONa & 25 \\
\hline $\mathbf{2 .}$ & $\boldsymbol{t}$-BuOK & $\mathbf{3 2}$ \\
\hline 3. & $\mathrm{KOH}$ & 10 \\
\hline 4. & $\mathrm{NaOH}$ & 25 \\
\hline 5. & $\mathrm{~K}_{3} \mathrm{PO}_{4}$ & 0 \\
\hline 6. & $\mathrm{Cs}_{2} \mathrm{CO}_{3}$ & 0 \\
\hline
\end{tabular}

Reaction condition: ${ }^{a}$-aminobutan-1-ol 1a $(0.25 \mathrm{mmol}), \mathbf{2 a}(0.5 \mathrm{mmol}), \mathrm{NiBr}_{2}(10 \mathrm{~mol} \%), \mathbf{L 1}(12 \mathrm{~mol} \%)$, Base (1.0 equiv.), toluene $\left(1.0 \mathrm{~mL}\right.$ ), Schenk tube under $\mathrm{N}_{2}$ atmosphere, $130{ }^{\circ} \mathrm{C}$ in oil bath, $36 \mathrm{~h}$ reaction time.

Table S3: Screening of solvents ${ }^{a}$

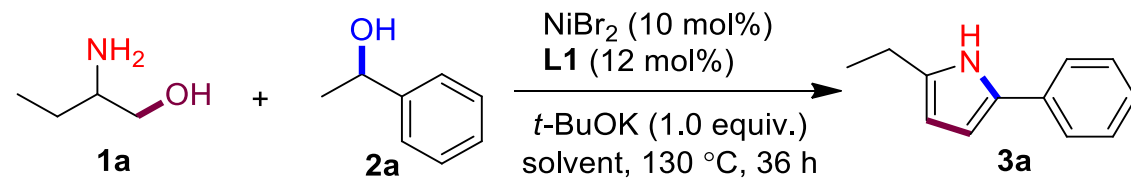

\begin{tabular}{|c|c|c|}
\hline \multicolumn{3}{|c|}{$\mathbf{2 a}$} \\
\hline Entry & Solvents & GC-MS Conversion 3a \\
\hline $\mathbf{1 .}$ & Toluene & $\mathbf{3 2}$ \\
\hline 2 & THF & 12 \\
\hline 3. & Xylene & 20 \\
\hline 4. & 2-methyl THF & 15 \\
\hline 5. & Dioxane & 13 \\
\hline 6. & $t$-AmOH & 12 \\
\hline
\end{tabular}

Reaction condition: ${ }^{a} 2$-aminobutan-1-ol 1a $(0.25 \mathrm{mmol}), \mathbf{2 a}(0.5 \mathrm{mmol}), \mathrm{NiBr}_{2}(10 \mathrm{~mol} \%), \mathbf{L 1}(12 \mathrm{~mol} \%)$, Base (1.0 equiv.), solvent $\left(1.0 \mathrm{~mL}\right.$ ), Schenk tube under $\mathrm{N}_{2}$ atmosphere, $130{ }^{\circ} \mathrm{C}$ in oil bath, $36 \mathrm{~h}$ reaction time. 
Table S4: Screening of base equivalents ${ }^{a}$

\begin{tabular}{|c|c|c|}
\hline Entry & Base equivalents & GC-MS Conversion 3a \\
\hline 1. & $t$-BuOK (1.0 equiv.) & 32 \\
\hline 2. & $t$-BuOK (1.5 equiv.) & 38 \\
\hline 3. & $\boldsymbol{t}$-BuOK (2.0 equiv.) & $\mathbf{4 2}$ \\
\hline 4. & - & $0 \%$ \\
\hline
\end{tabular}

Reaction condition: ${ }^{a}$-aminobutan-1-ol 1a $(0.25 \mathrm{mmol}), \mathbf{2 a}(0.5 \mathrm{mmol}), \mathrm{NiBr}_{2}(10 \mathrm{~mol} \%), \mathbf{L 1}(12 \mathrm{~mol} \%)$, Base (X equiv.), toluene (1.0 mL), Schenk tube under $\mathrm{N}_{2}$ atmosphere, $130{ }^{\circ} \mathrm{C}$ in oil bath, $36 \mathrm{~h}$ reaction time.

Table S5: Screening of amino alcohol and alcohol equivalents ${ }^{a}$<smiles>CCC(N)CO</smiles>

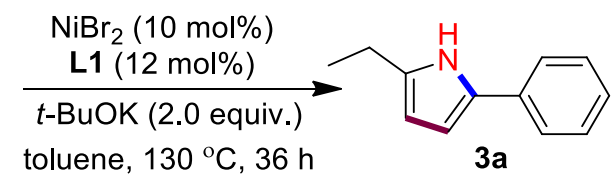

\begin{tabular}{|c|c|c|c|}
\hline Entry & 1a X mmol & 2a Y mmol & GC-MS Conversion 3a \\
\hline $\mathbf{1 .}$ & $\mathbf{0 . 5}$ & $\mathbf{1 . 0}$ & $\mathbf{4 2}$ \\
\hline 2. & 0.5 & 0.5 & 32 \\
\hline 3. & 0.75 & 0.5 & 28 \\
\hline 4. & 1.0 & 0.5 & 26 \\
\hline
\end{tabular}

Reaction condition: ${ }^{a}$-aminobutan-1-ol 1a (X mmol), 2a (Y mmol), $\mathrm{NiBr}_{2}$ (10 mol\%), L1 (12 mol\%), $t$-BuOK (2.0 equiv.), toluene $\left(1.0 \mathrm{~mL}\right.$ ), Schenk tube under $\mathrm{N}_{2}$ atmosphere, $130{ }^{\circ} \mathrm{C}$ in oil bath, $36 \mathrm{~h}$ reaction time.

Table S6: Screening of catalyst/ligand loading ${ }^{a}$

\begin{tabular}{|c|c|c|c|}
\hline Entry & Catalyst loading & Ligand Loading & GC-MS Conversion 3a \\
\hline $\mathbf{1 .}$ & $\mathbf{N i B r} \mathbf{2}(\mathbf{1 0} \mathbf{~ m o l \% )}$ & $\mathbf{L 1}(\mathbf{1 2} \mathbf{~ m o l} \%)$ & $\mathbf{4 2}$ \\
\hline 2. & $\operatorname{NiBr}_{2}(7.5 \mathrm{~mol} \%)$ & $\mathbf{L 1}(9 \mathrm{~mol} \%)$ & 31 \\
\hline 3. & $\operatorname{NiBr}_{2}(5.0 \mathrm{~mol} \%)$ & $\mathbf{L 1}(6 \mathrm{~mol} \%)$ & 24 \\
\hline 5. & - & - & 0 \\
\hline
\end{tabular}

Reaction condition: ${ }^{a}$-aminobutan-1-ol 1a $(0.25 \mathrm{mmol}), \mathbf{2 a}(0.5 \mathrm{mmol}), \mathrm{NiBr}_{2}(\mathrm{X} \mathrm{mol} \%), \mathbf{L 1}(\mathrm{X} \mathrm{mol} \%), t$-BuOK (2.0 equiv.), toluene $\left(1.0 \mathrm{~mL}\right.$ ), Schenk tube under $\mathrm{N}_{2}$ atmosphere, $130{ }^{\circ} \mathrm{C}$ in oil bath, $36 \mathrm{~h}$ reaction time.

Table S7: Screening of temperature ${ }^{a}$

\begin{tabular}{|c|c|c|}
\hline Entry & Temp. $^{\circ} \mathrm{C}$ & GC-MS Conversion 3a \\
\hline 1. & 130 & 42 \\
\hline 2. & 140 & 45 \\
\hline $\mathbf{3 .}$ & $\mathbf{1 5 0}$ & $\mathbf{5 3}(\mathbf{4 8})^{\boldsymbol{c}}$ \\
\hline
\end{tabular}

Reaction condition: ${ }^{a}$-aminobutan-1-ol 1a $(0.25 \mathrm{mmol}), \mathbf{2 a}(0.5 \mathrm{mmol}), \mathrm{NiBr}_{2}(10 \mathrm{~mol} \%), \mathbf{L 1}(12 \mathrm{~mol} \%), t$-BuOK (2.0 equiv.), toluene $\left(1.0 \mathrm{~mL}\right.$ ), Schenk tube under $\mathrm{N}_{2}$ atmosphere, $\mathrm{X}{ }^{\circ} \mathrm{C}$ in oil bath, $36 \mathrm{~h}$ reaction time. ${ }^{b}$ Isolated yield. ${ }^{c}$ Reaction was carried out in high pressure reactor. 


\section{[1.2] Mechanistic studies:}

\section{Scheme S1: Intermolecular Cyclization to pyrrole}

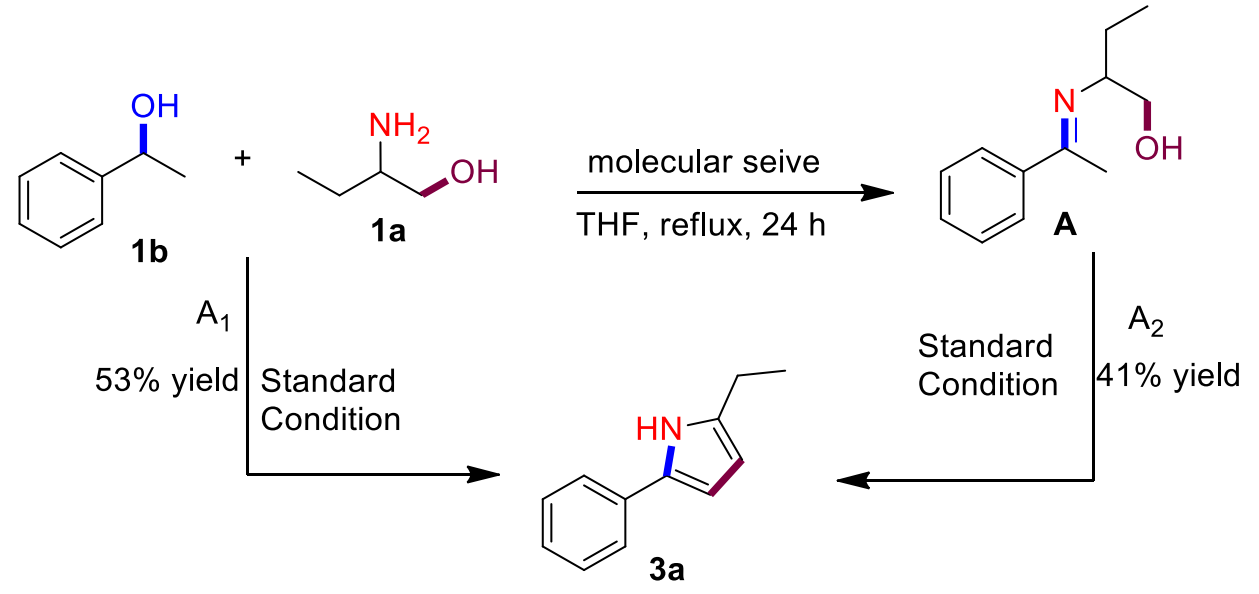

These experiments support the observations for the involvement of intermediate A during the intermolecular cyclization to pyrrole. As in both cases, almost equivalent amount of pyrrole was formed.

\section{Scheme S2: Reactions which contradict Pathway II:}

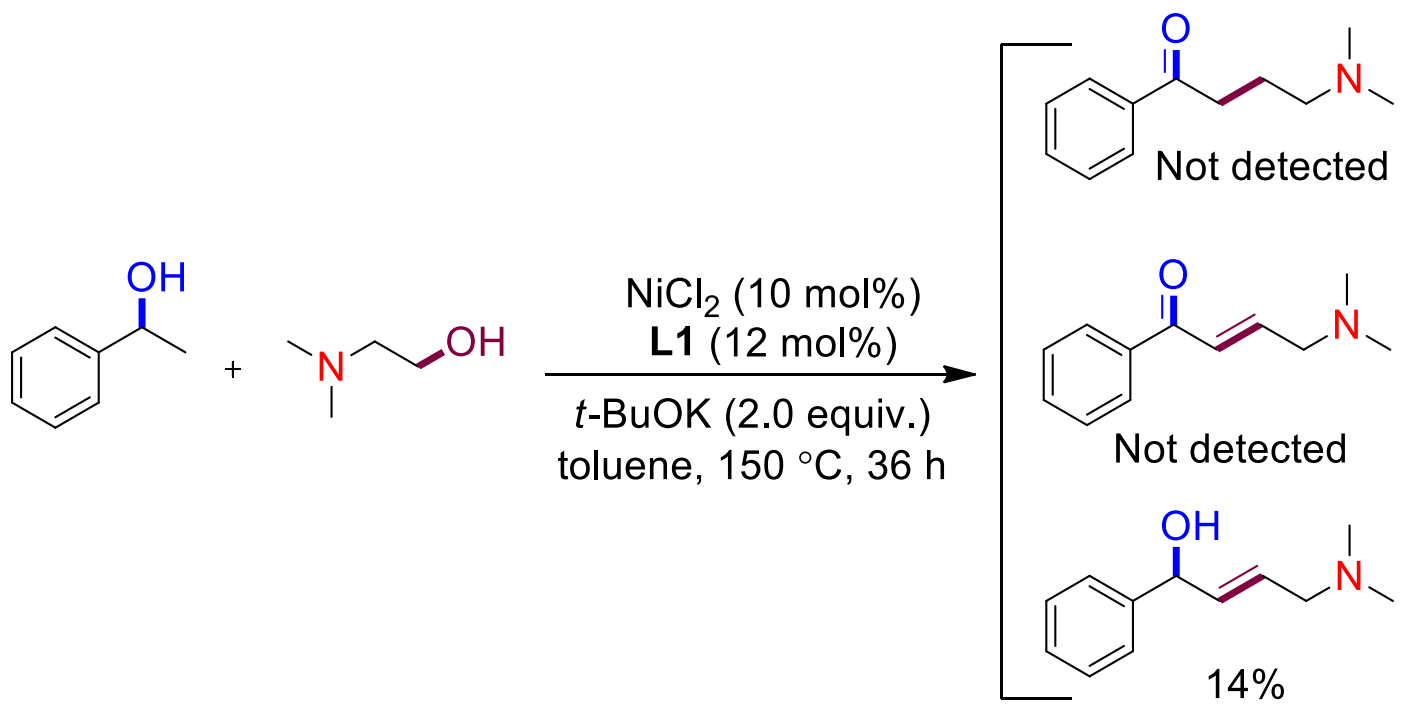

The reaction between 1-phenylethanol 2a and the $N$-protected amino alcohol under optimized reaction conditions, only $14 \%$ of the $\alpha$-alkylation product was observe. This experiment indicated that under the standard reaction conditions, $\alpha$-alkylation is quite slow and unlikely to be the first reaction step (pathway II, Scheme S1) in the pyrrole syntheses. 


\section{[1.3] Deuterium incorporation experiments:}

\section{Scheme S3:}
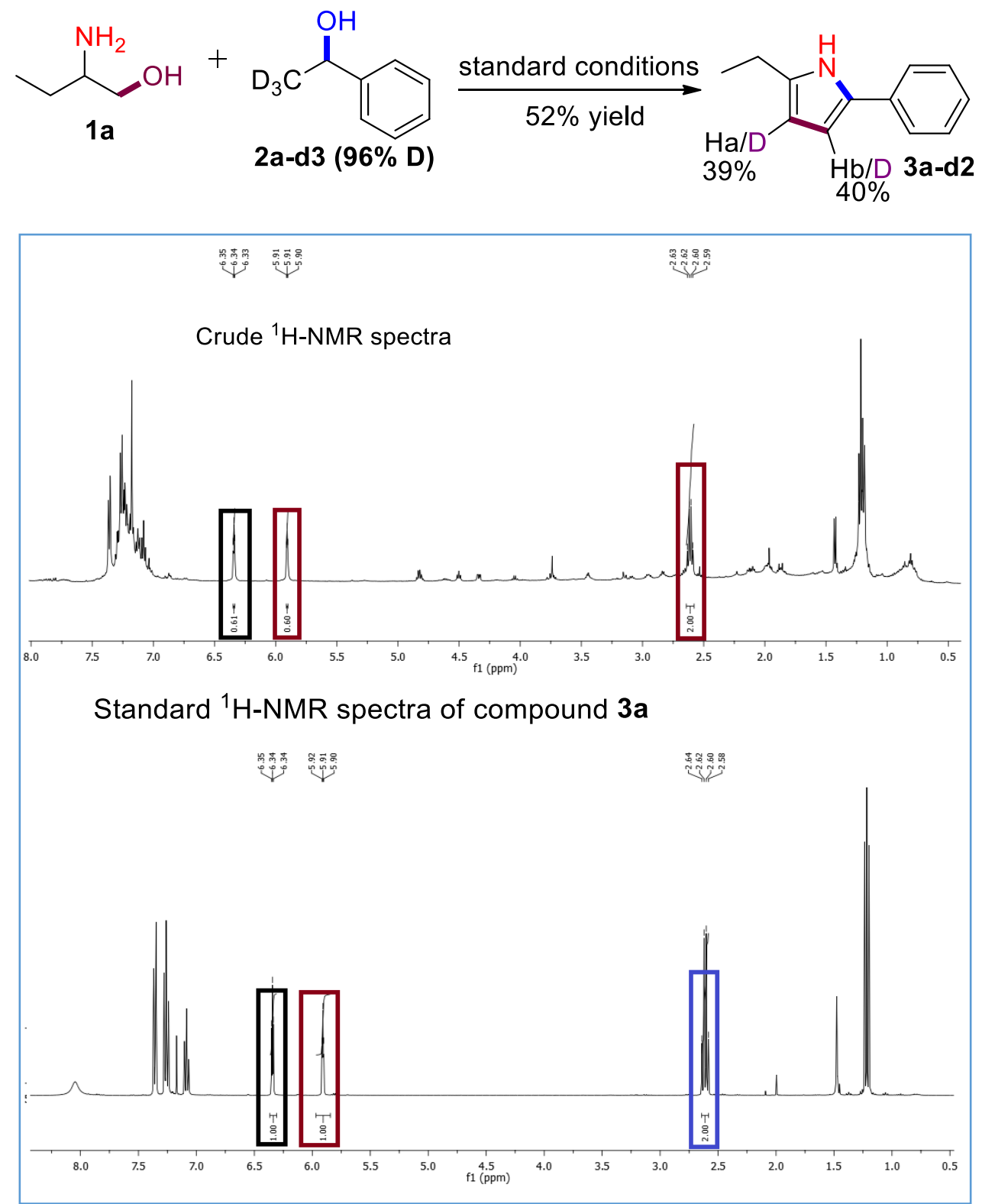

Conversion was calculated by ${ }^{1} \mathrm{H}-\mathrm{NMR}$ integration value

\begin{tabular}{|l|c|c|c|}
\hline & & $\begin{array}{c}\text { Deuterium } \\
\text { incorporation in } \\
\text { a position }\end{array}$ & $\begin{array}{c}\text { Deuterium } \\
\text { incorporation in } \\
\text { b position }\end{array}$ \\
\hline Signal $\delta \mathrm{ppm}$ & $2.70(2 \mathrm{H})$ & $6.41(1 \mathrm{H})$ & $5.98(1 \mathrm{H})$ \\
\hline Integral Value & 2.0 & 0.61 & 0.60 \\
\hline $\begin{array}{l}\text { Calculated } \\
\text { ratio }\end{array}$ & & $\{(1-0.65)\} \times 100=$ & $\{(1-0.65)\} \times 100=$ \\
$\mathbf{4 0 \%}$
\end{tabular}




\section{Scheme S4:}<smiles>CCC(N)CO</smiles>

$1 \mathrm{a}$
$\mathrm{H}_{3} \mathrm{C}$

2a-d1 (96\% D) $\underset{58 \% \text { standard conditions }}{\longrightarrow}$ $58 \%$ yield

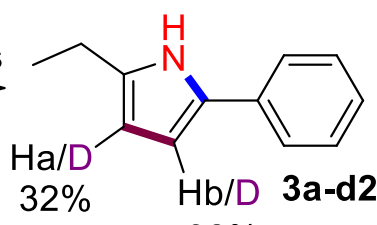

$32 \%$

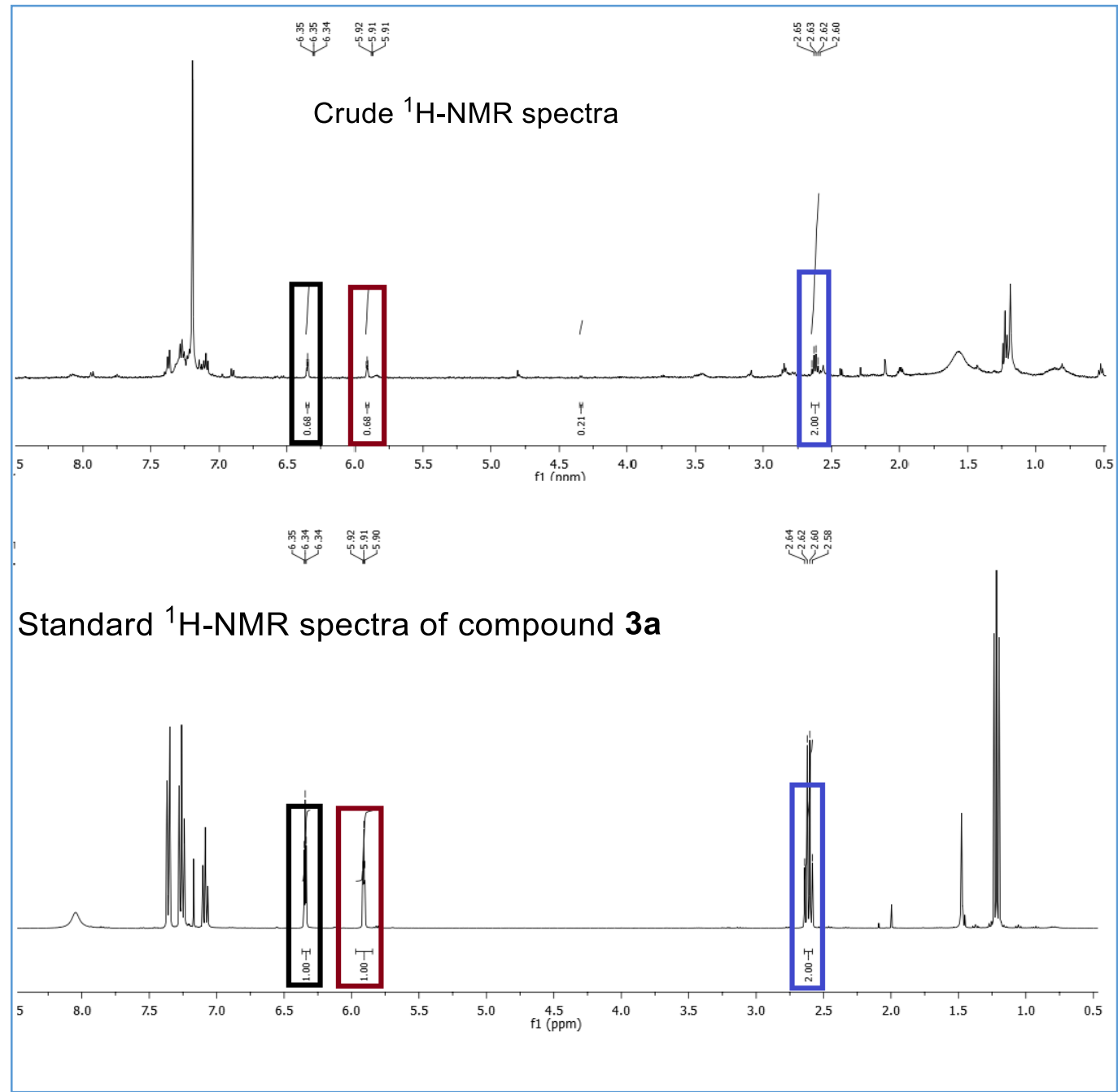

Conversion was calculated by ${ }^{1} \mathrm{H}-\mathrm{NMR}$ integration value

\begin{tabular}{|l|c|c|c|}
\hline & & $\begin{array}{c}\text { Deuterium } \\
\text { incorporation in } \\
\text { a position }\end{array}$ & $\begin{array}{c}\text { Deuterium } \\
\text { incorporation in } \\
\text { b position }\end{array}$ \\
\hline Signal $\delta \mathrm{ppm}$ & $2.70(2 \mathrm{H})$ & $6.41(1 \mathrm{H})$ & $5.98(1 \mathrm{H})$ \\
\hline Integral Value & 2.0 & 0.68 & 0.68 \\
\hline $\begin{array}{l}\text { Calculated } \\
\text { ratio }\end{array}$ & & $\begin{array}{c}\{(1-0.71)\} \times 100= \\
\mathbf{3 2 \%}\end{array}$ & $\begin{array}{c}\{(1-0.70)\} \times 100= \\
\mathbf{3 2 \%}\end{array}$ \\
\hline
\end{tabular}


Scheme S5: Quantitative determination of hydrogen gas produced in the reaction In a $10 \mathrm{~mL}$ oven dried Schlenk tube, 2-aminobutan-1-ol $\mathbf{1 a}(0.25 \mathrm{mmol}), \mathrm{NiBr}_{2}(10 \mathrm{~mol} \%)$, Phen L1 (12 mol\%), 1-phenylethanol $2 \mathbf{a}(0.5 \mathrm{mmol})$ and $t$-BuOK (2.0 equiv.) were added followed by toluene $1.0 \mathrm{~mL}$ and connected to the gas burette as shown in below figure. Then the reaction mixture was heated at $150{ }^{\circ} \mathrm{C}$ until the production of hydrogen gas ceased. The procedure was repeated three times to get concordant reading.

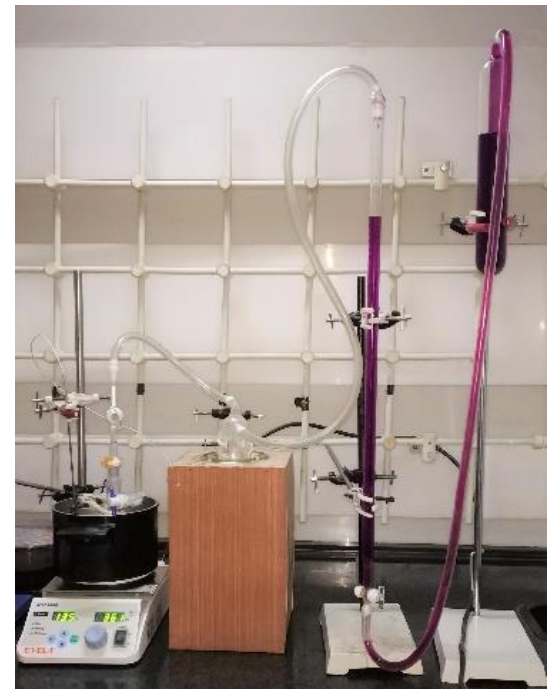

Total volume of water displaced, $\mathrm{V}=0.017 \mathrm{~L}$

Vapor pressure of water at $298 \mathrm{~K}, \mathrm{P}_{\mathrm{H} 2 \mathrm{O}}=23.7695$ Torr

Atmospheric pressure at $298 \mathrm{~K}, \mathrm{P}_{\mathrm{atm}}=758.3124$ Torr

Pressure of $\mathrm{H}_{2}$ gas, $\mathrm{P}_{\mathrm{H} 2}=\mathrm{P}_{\mathrm{atm}}-\mathrm{P}_{\mathrm{H} 2 \mathrm{O}}=(758.3124-23.7695)$ Torr $=734.5429$ Torr

$$
\begin{aligned}
& \mathrm{P}_{\mathrm{H} 2} * \mathrm{~V}=\mathrm{nH}_{2} * \mathrm{R} * \mathrm{~T} \\
& \mathrm{nH}_{2}=\mathrm{P}_{\mathrm{H} 2} * \mathrm{~V} / \mathrm{R} * \mathrm{~T} \\
&= 734.5429 \text { Torr } * 0.017 \mathrm{~L} / 62.3635 \mathrm{~L} \mathrm{Torr}^{-1} \mathrm{~mol}^{-1} * 298 \mathrm{~K} \\
&=20.5672 / 18584.323 \\
&=0.00067 \mathrm{~m}+\mathrm{ol} \\
& \approx 0.67 \mathrm{mmol}
\end{aligned}
$$


Scheme S6: Detection of water in reaction mixture by ${ }^{1} \mathrm{H}-\mathrm{NMR}$<smiles>CCC(N)CO</smiles>

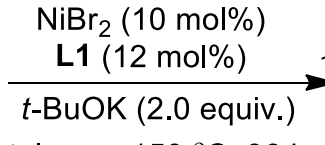

toluene, $150{ }^{\circ} \mathrm{C}, 36 \mathrm{~h}$
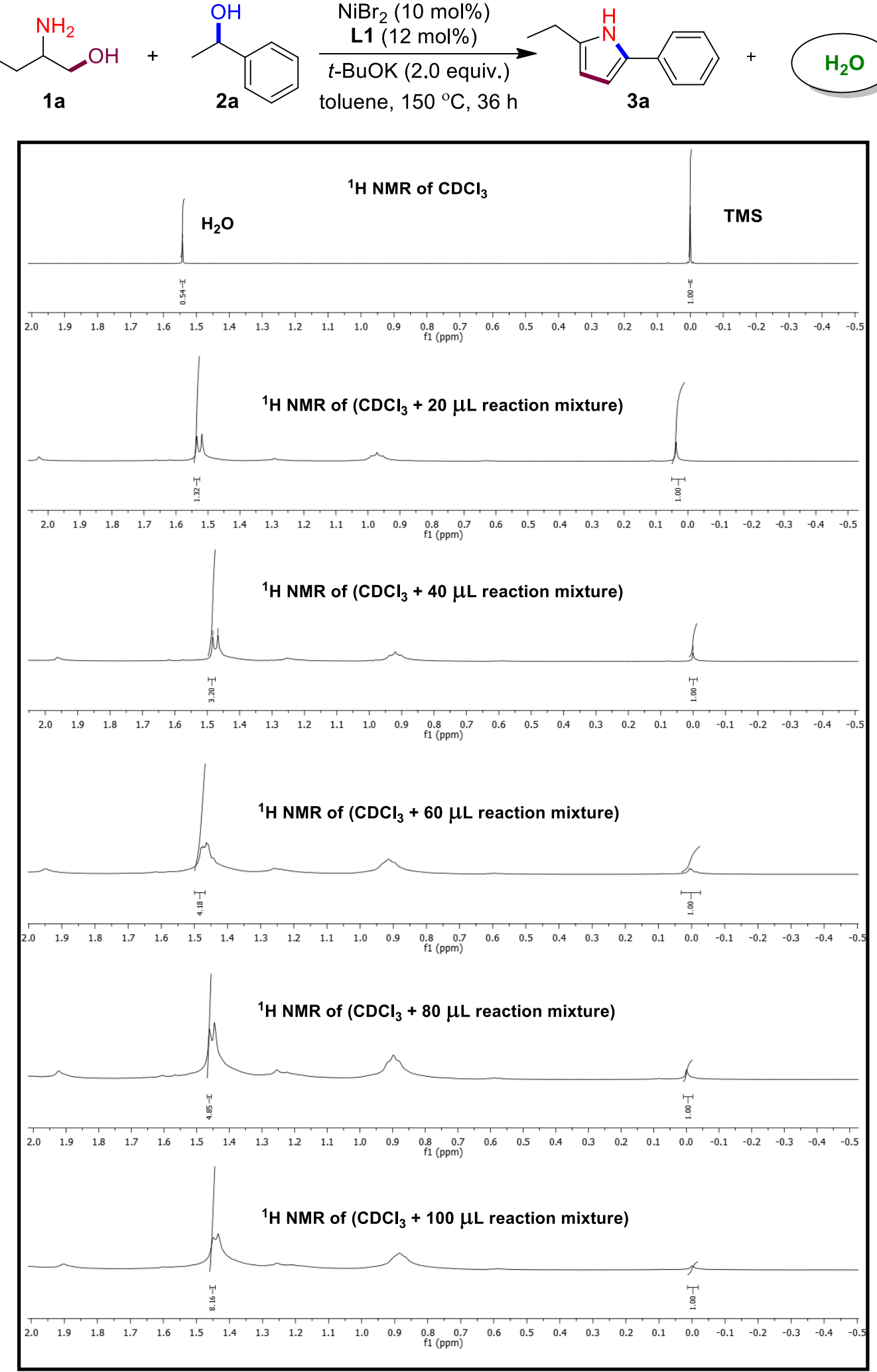

In a $15 \mathrm{~mL}$ oven dried Schlenk tube, 2-aminobutan-1-ol $\mathbf{1 a}(0.25 \mathrm{mmol}), \mathrm{NiBr}_{2}(10 \mathrm{~mol} \%)$, 1,10-phenanthroline L1 (12 mol\%), 1-phenylethanol 2a (0.5 mmol) and $t$-BuOK (2.0 equiv.) 
were added followed by toluene (dry) $1.0 \mathrm{~mL}$ under an atmosphere of $\mathrm{N}_{2}$ and the reaction mixture was heated at $150{ }^{\circ} \mathrm{C}$ for $36 \mathrm{~h}$ in a closed system. Then the reaction mixture was cooled to room temperature. Initially ${ }^{1} \mathrm{H} \mathrm{NMR}$ of $\mathrm{CDCl}_{3}$ was measured and 0.58:1 ratio of $\mathrm{H}_{2} \mathrm{O}$ and TMS was found. Afterwards $20 \mu \mathrm{L}$ of reaction mixture was added to the NMR tube and ${ }^{1} \mathrm{H}-$ NMR was measured which shows increment in the ratio of $\mathrm{H}_{2} \mathrm{O}$. Further addition of reaction mixture shows enhancement in the ratio of $\mathrm{H}_{2} \mathrm{O}$ which proves that water was produced in the reaction. 
[1.4] Copies of ${ }^{1} \mathrm{H}$ and ${ }^{13} \mathrm{C}$ NMR spectra for selected compounds.
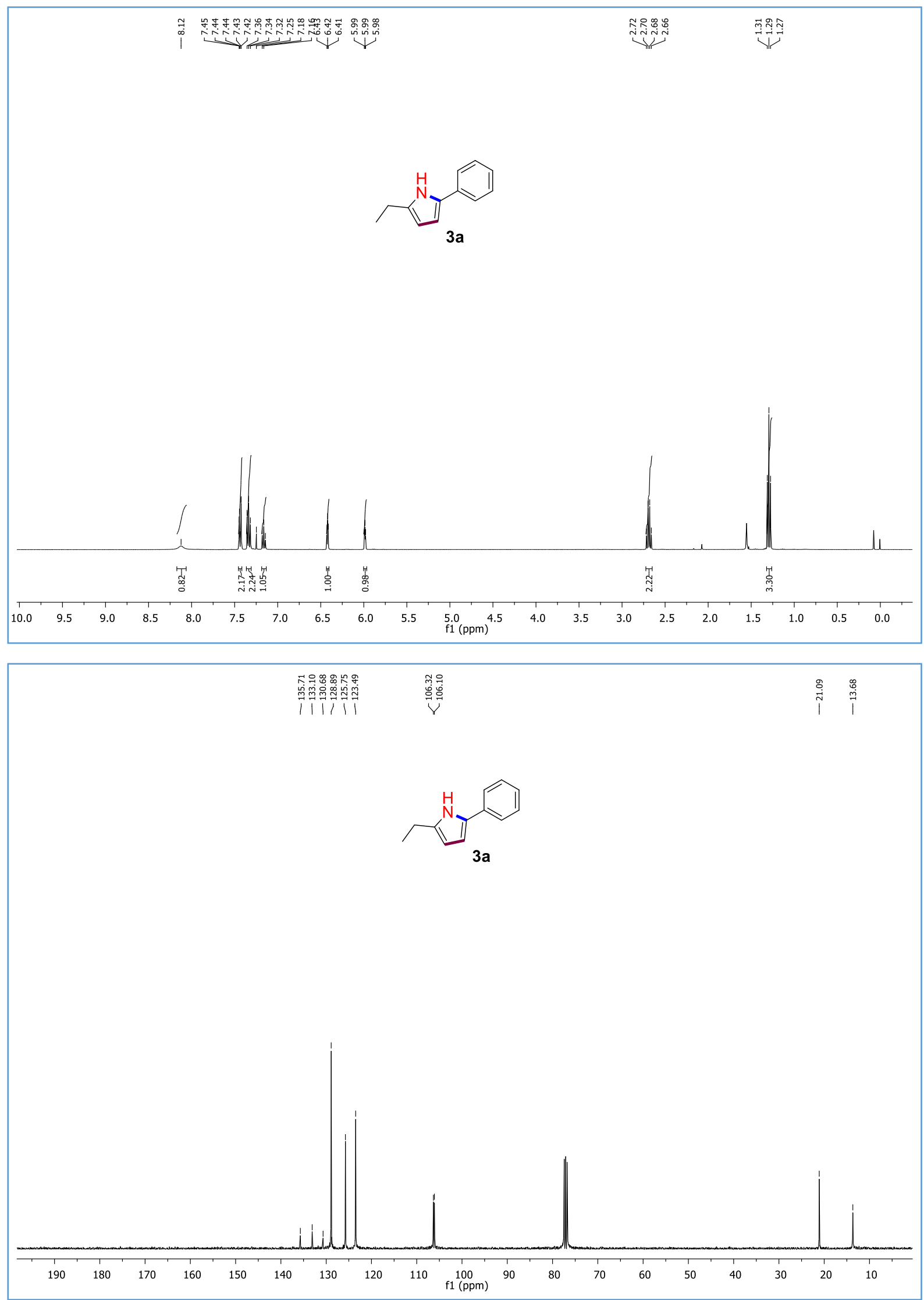

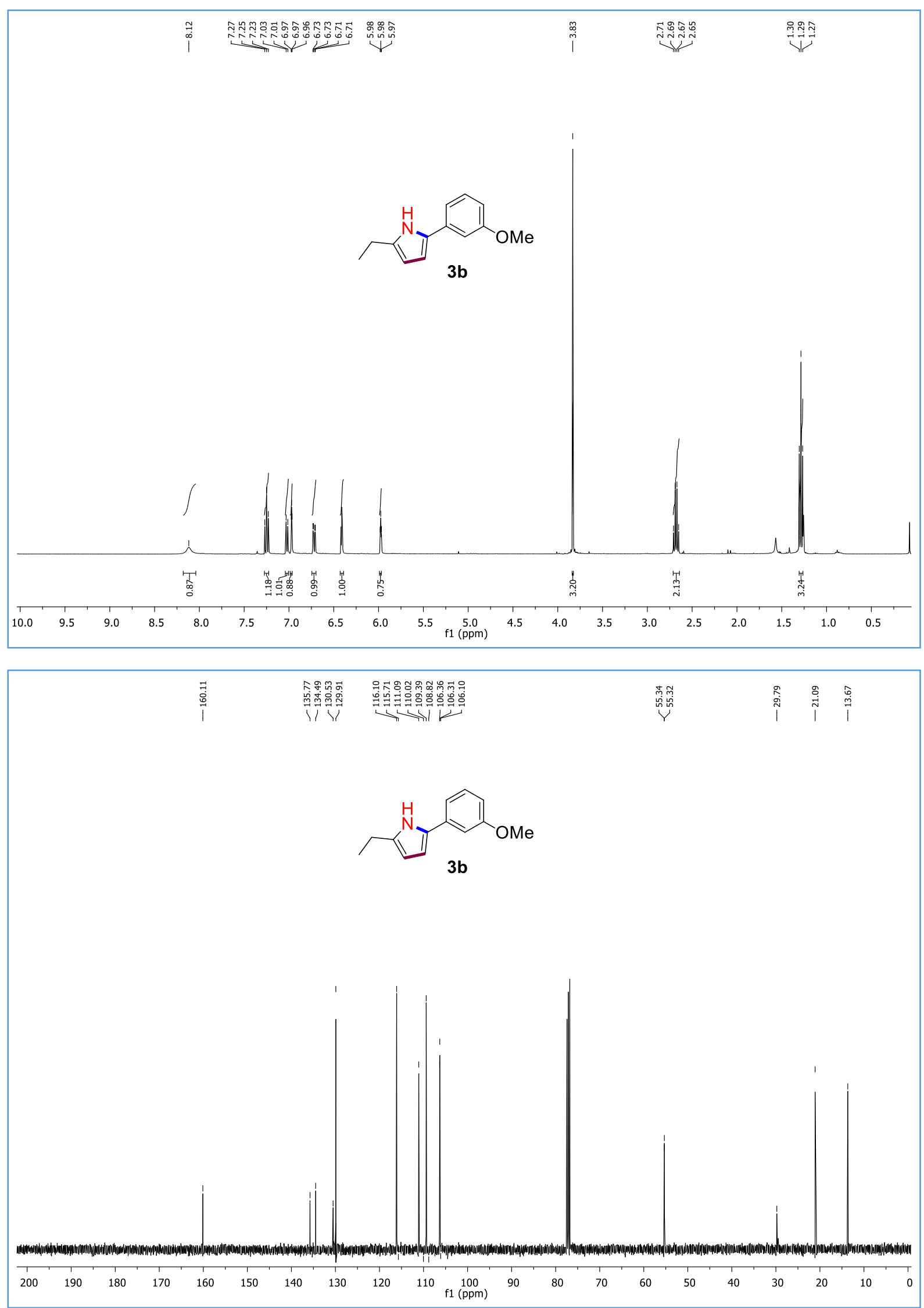

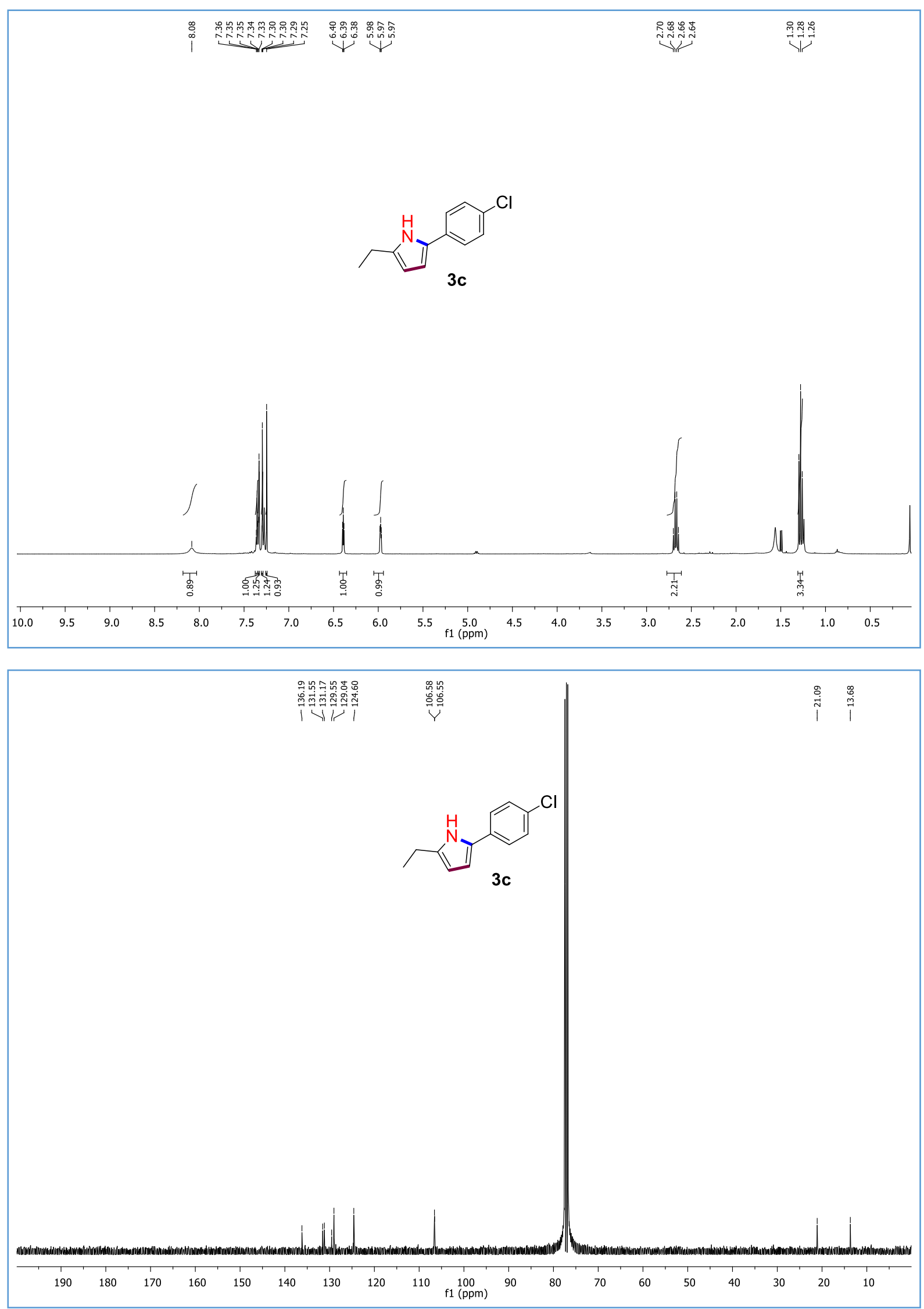

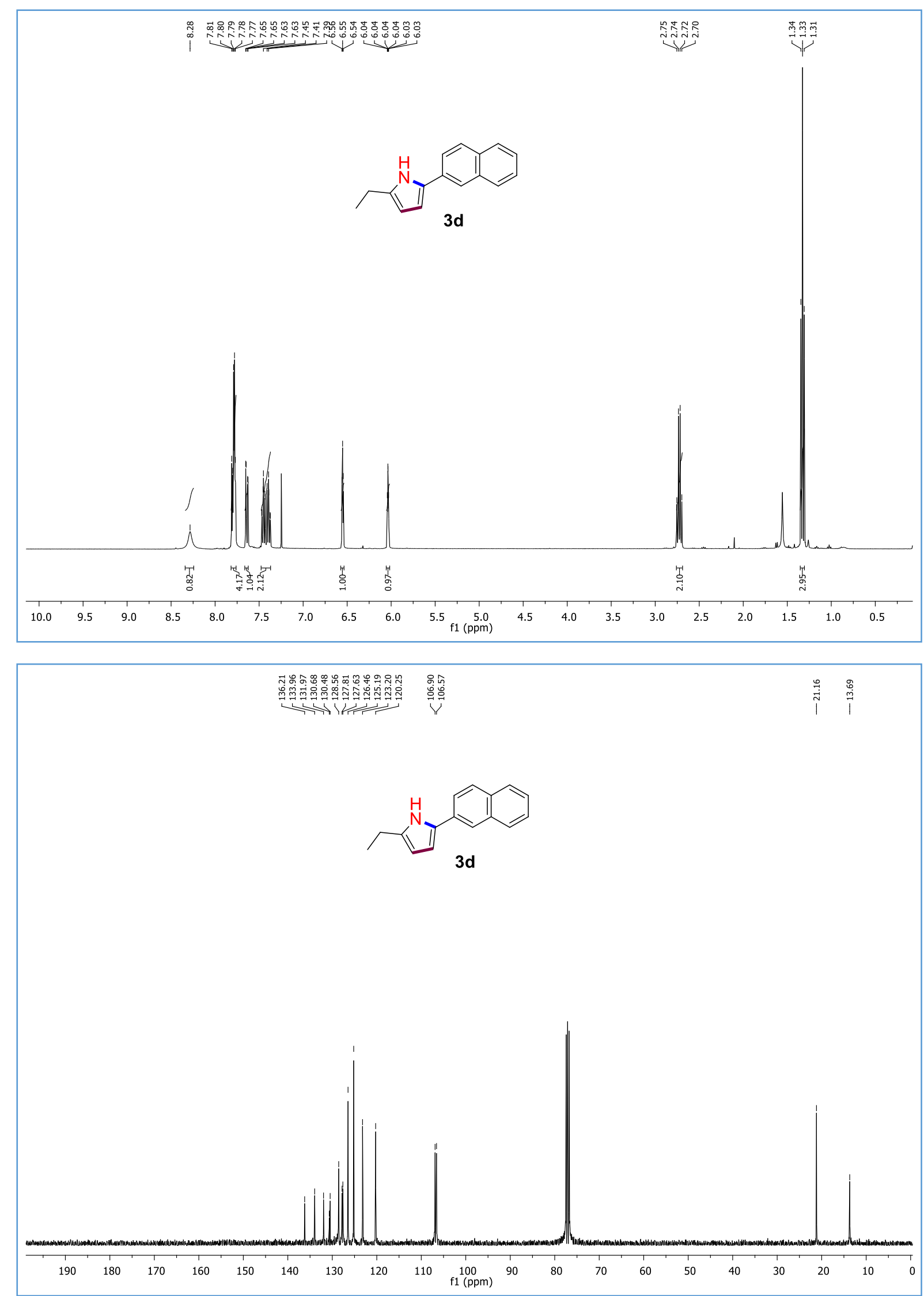

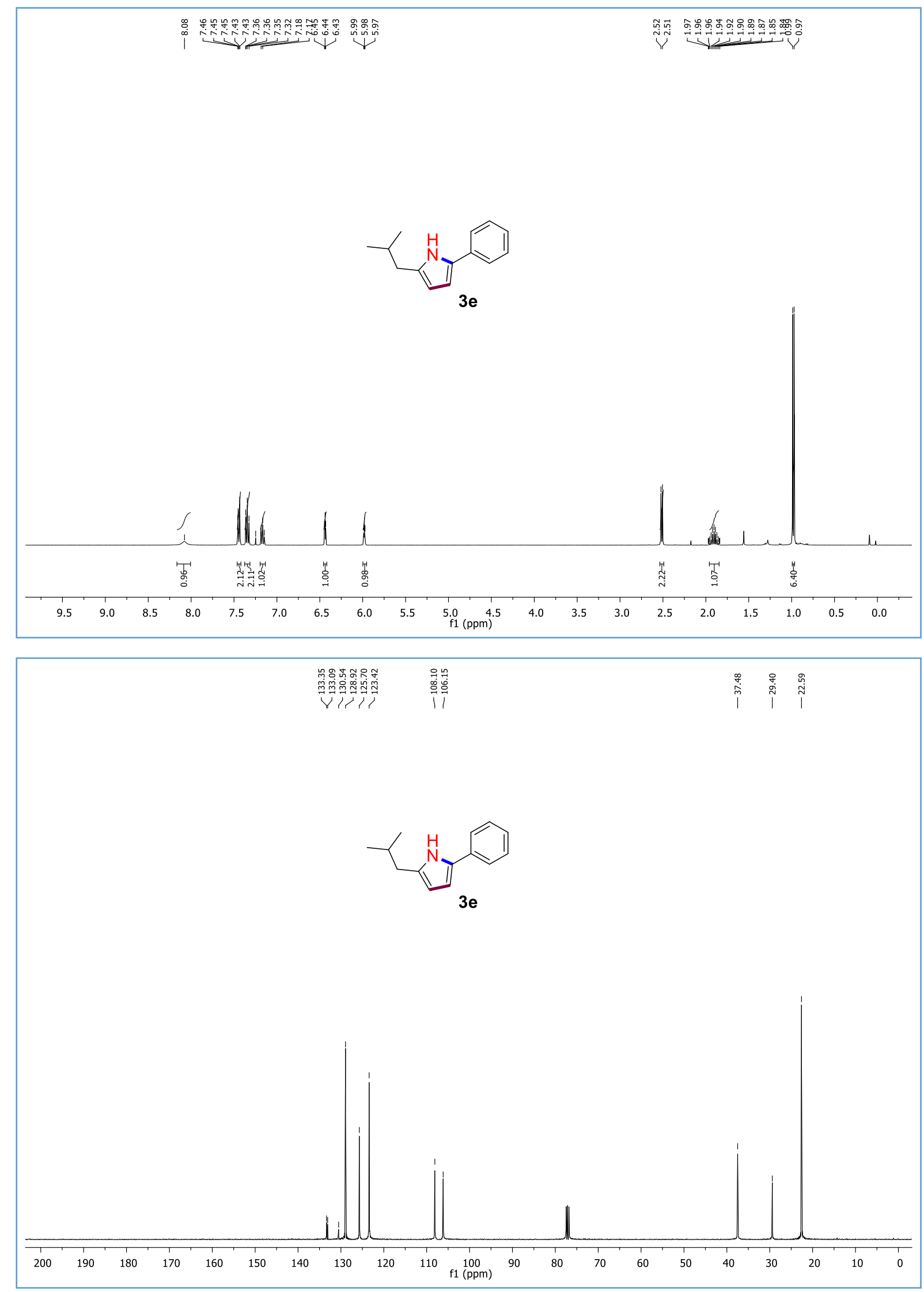

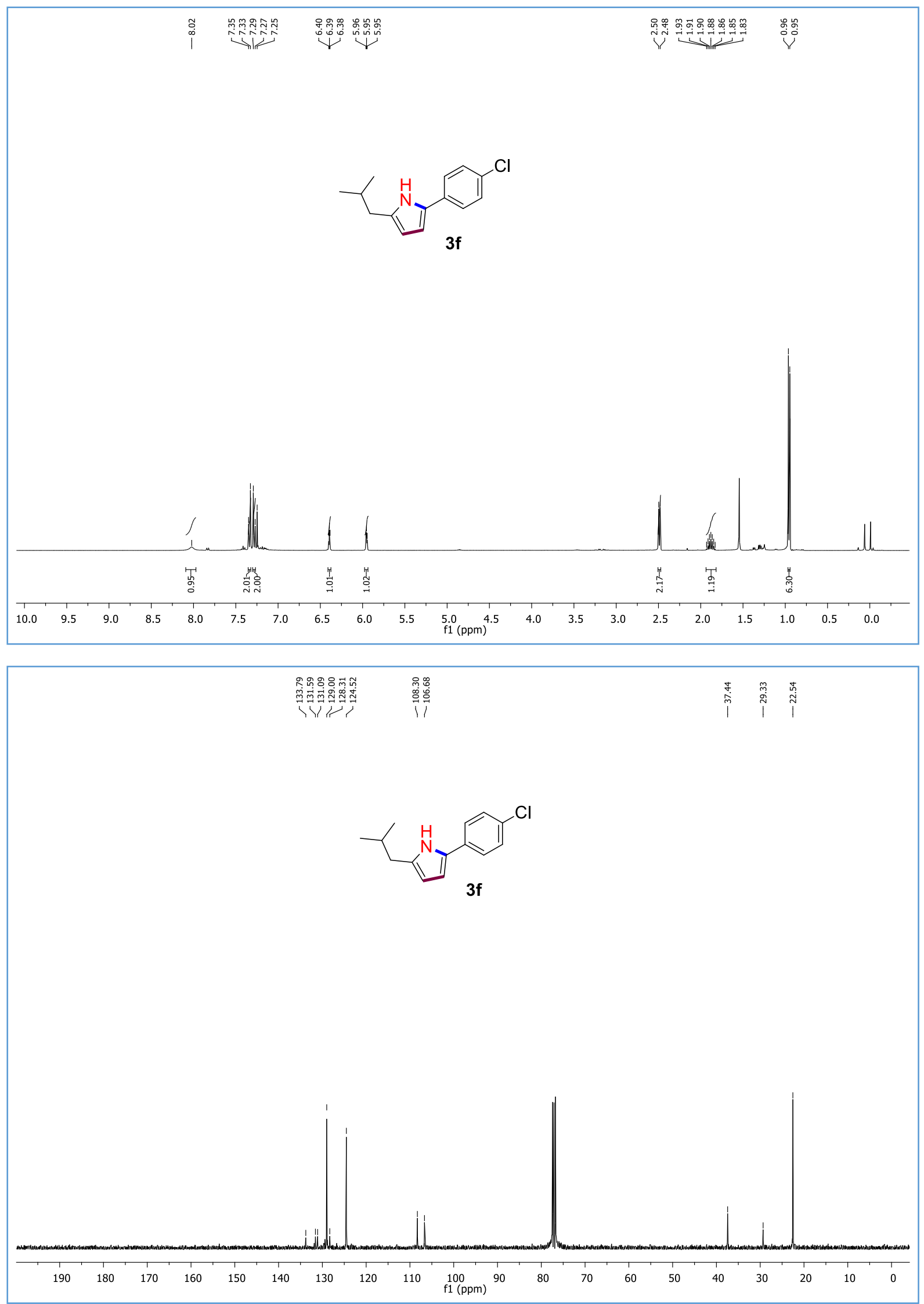

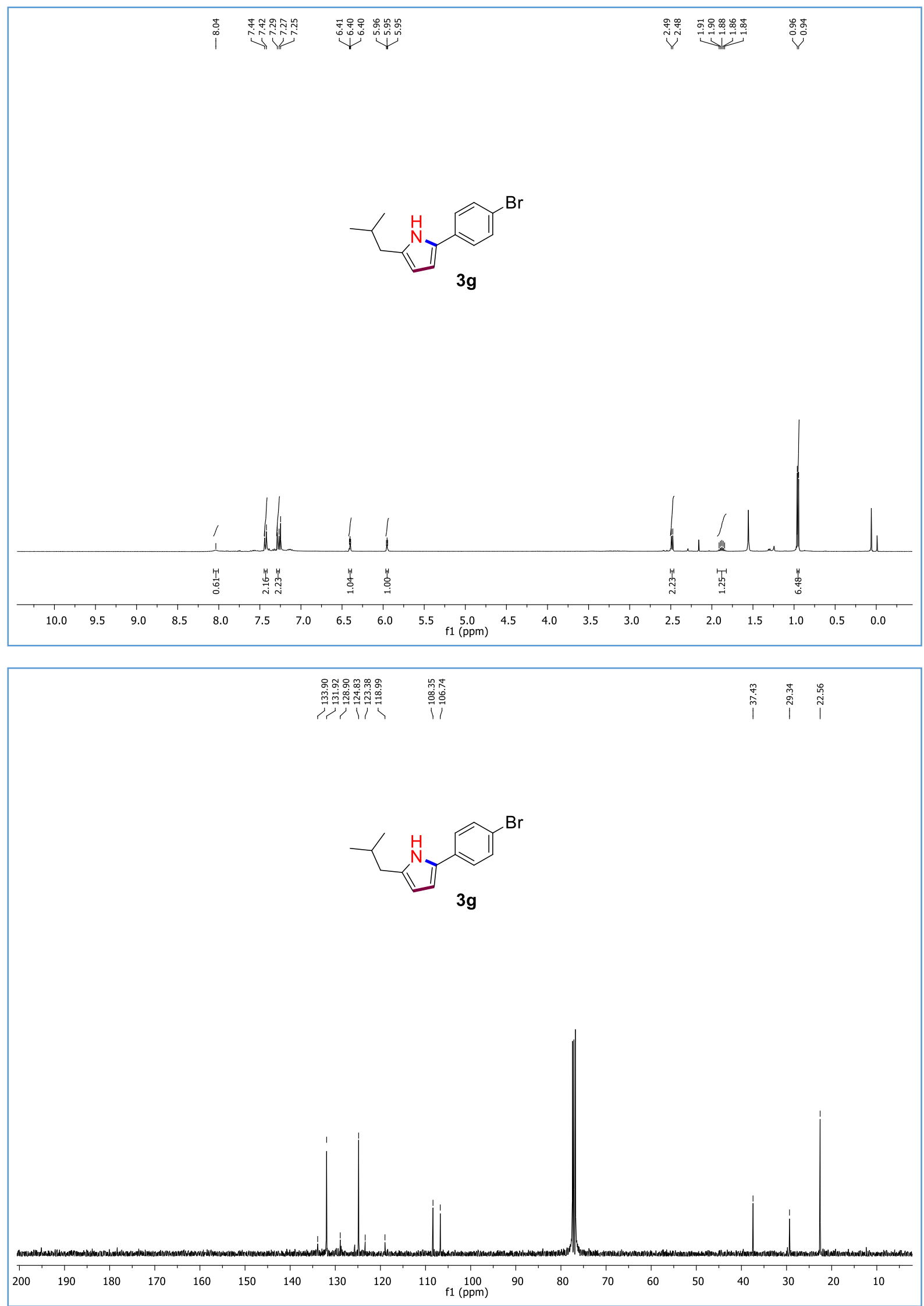

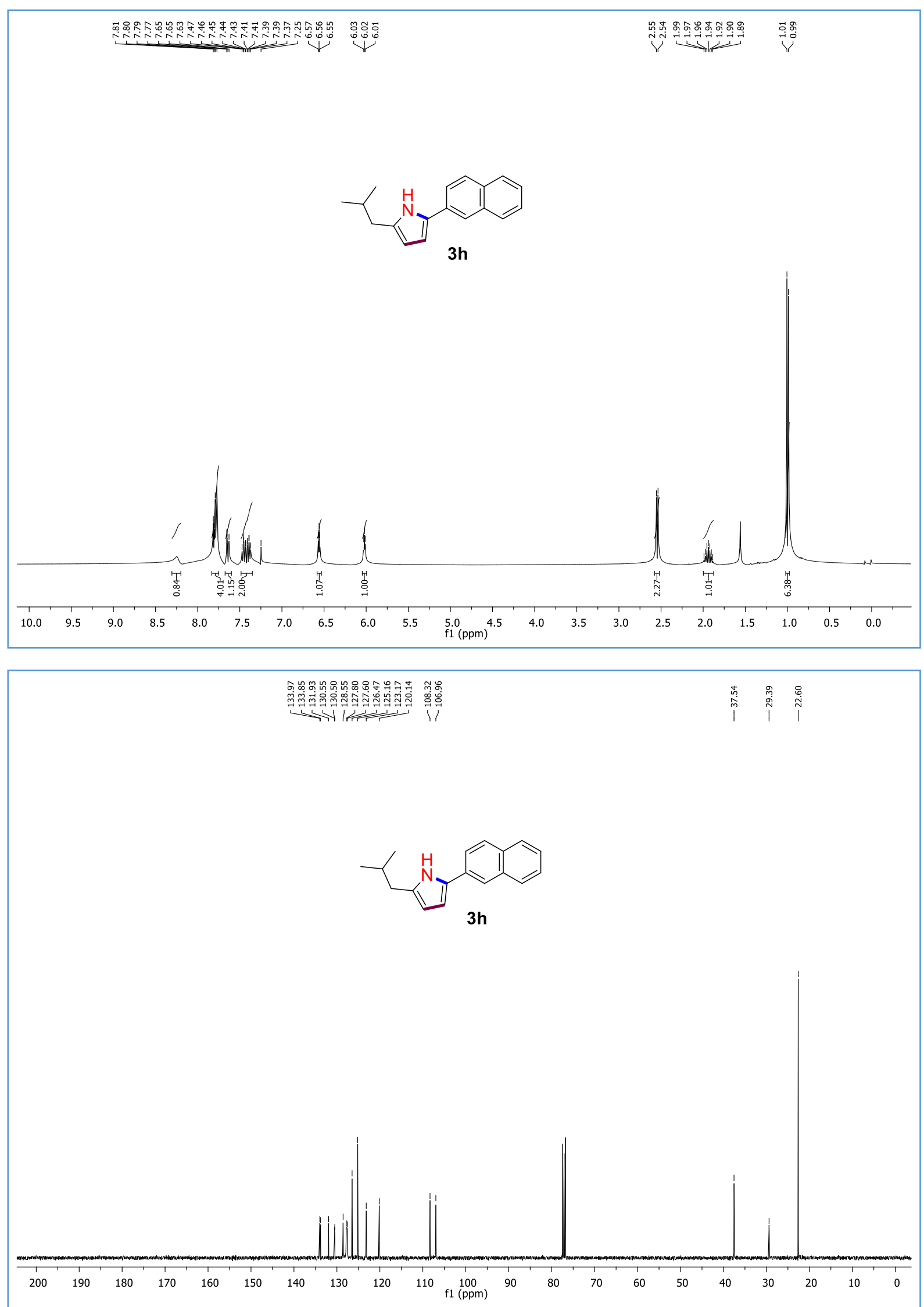

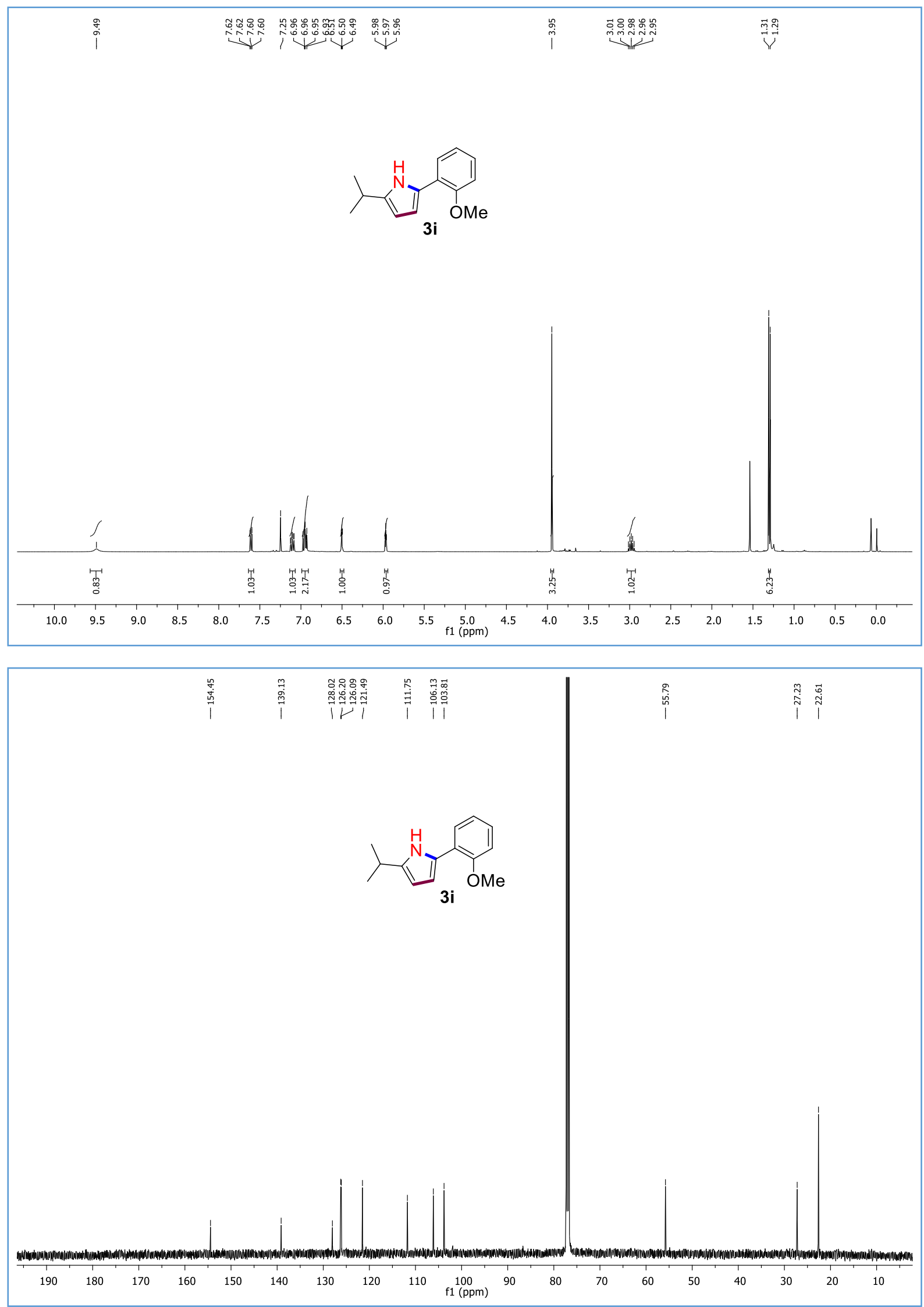

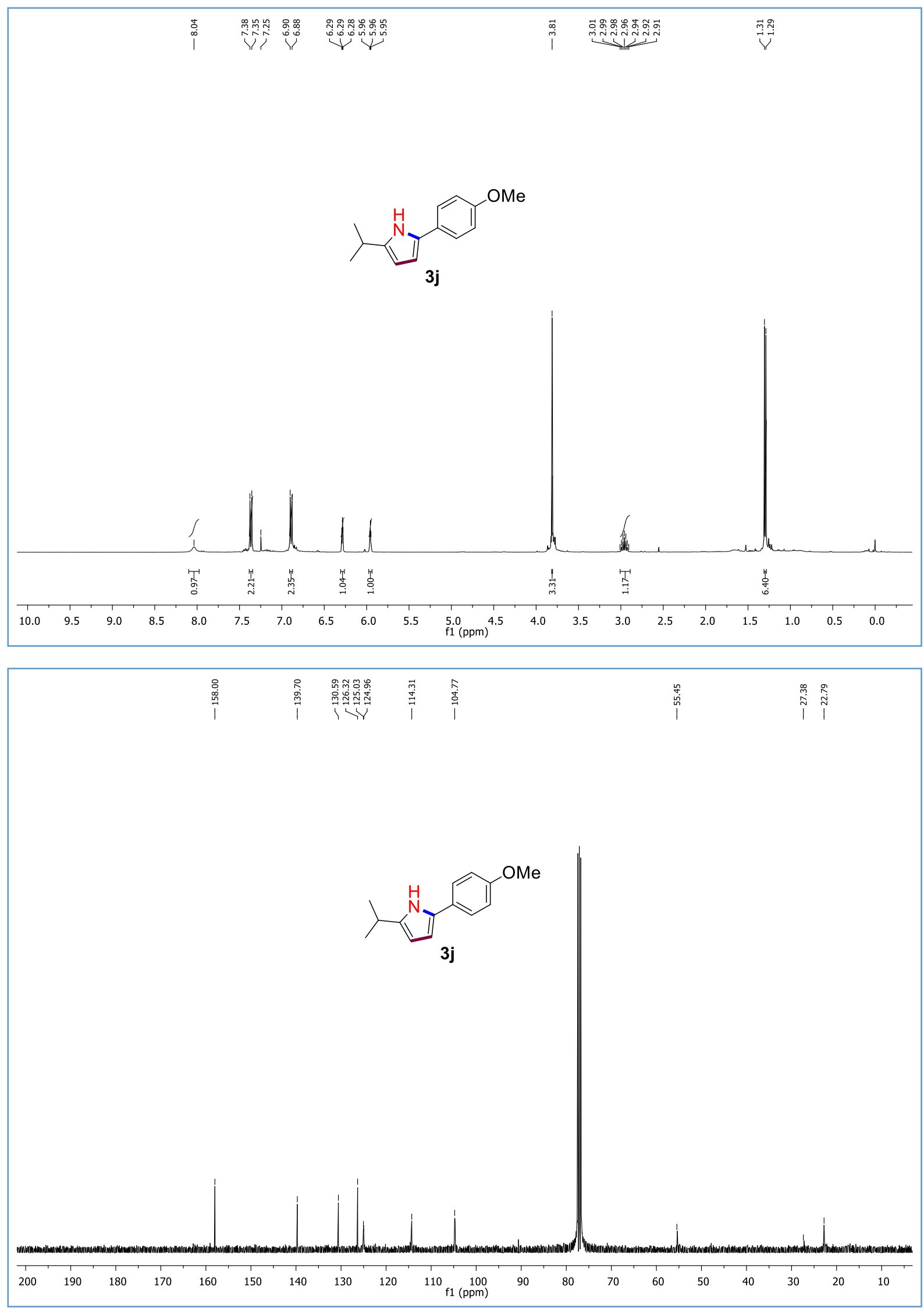


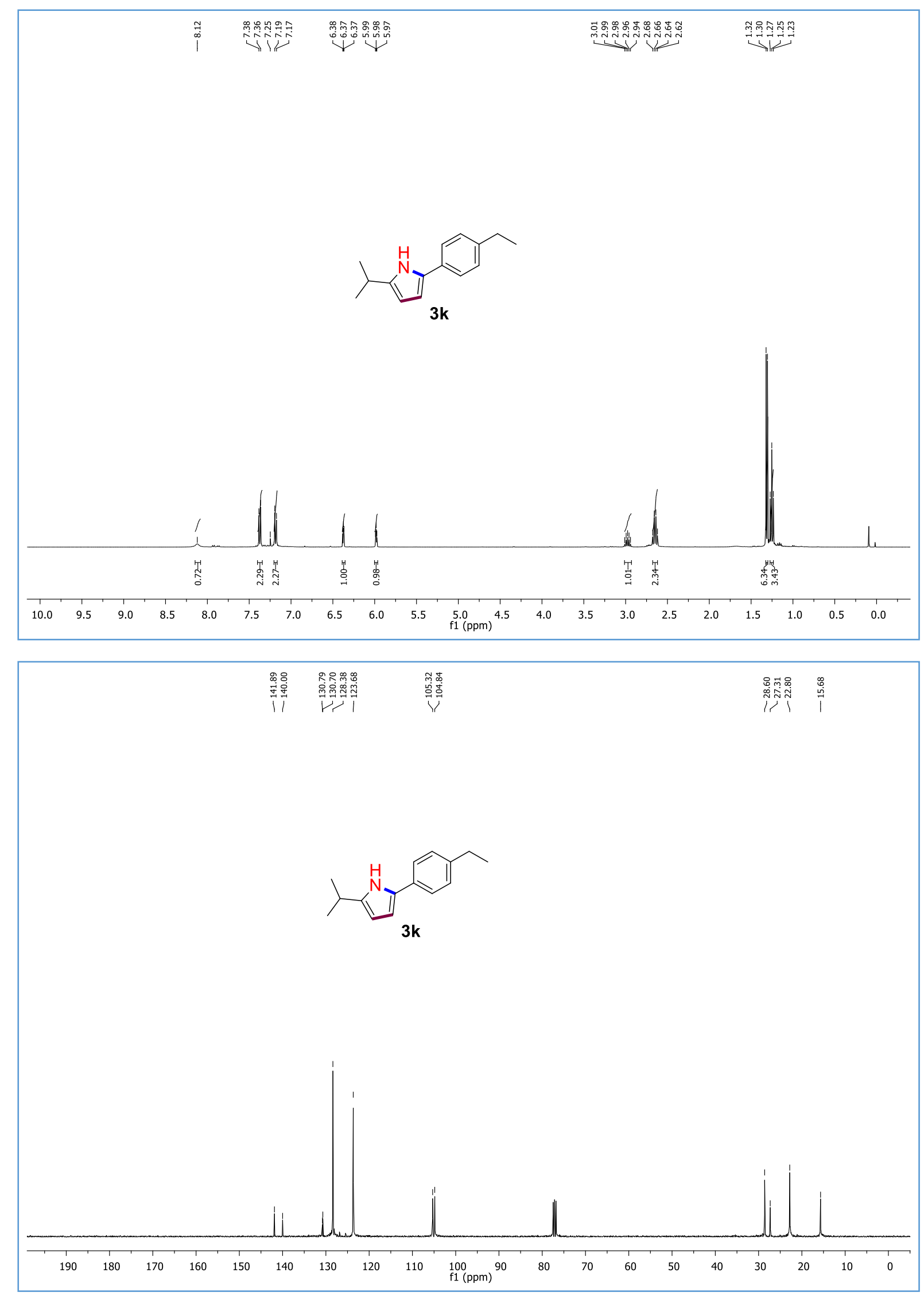

S20 

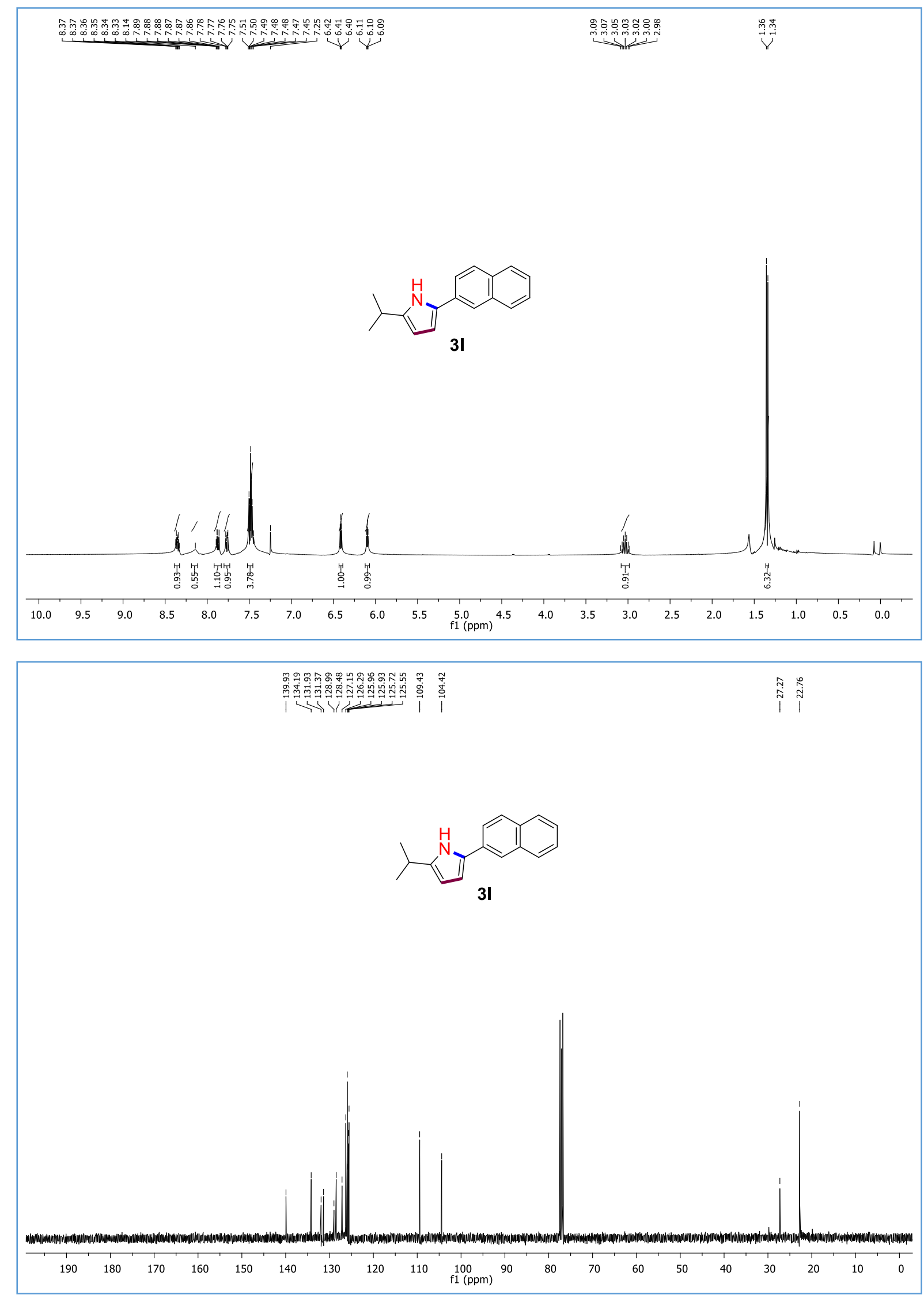

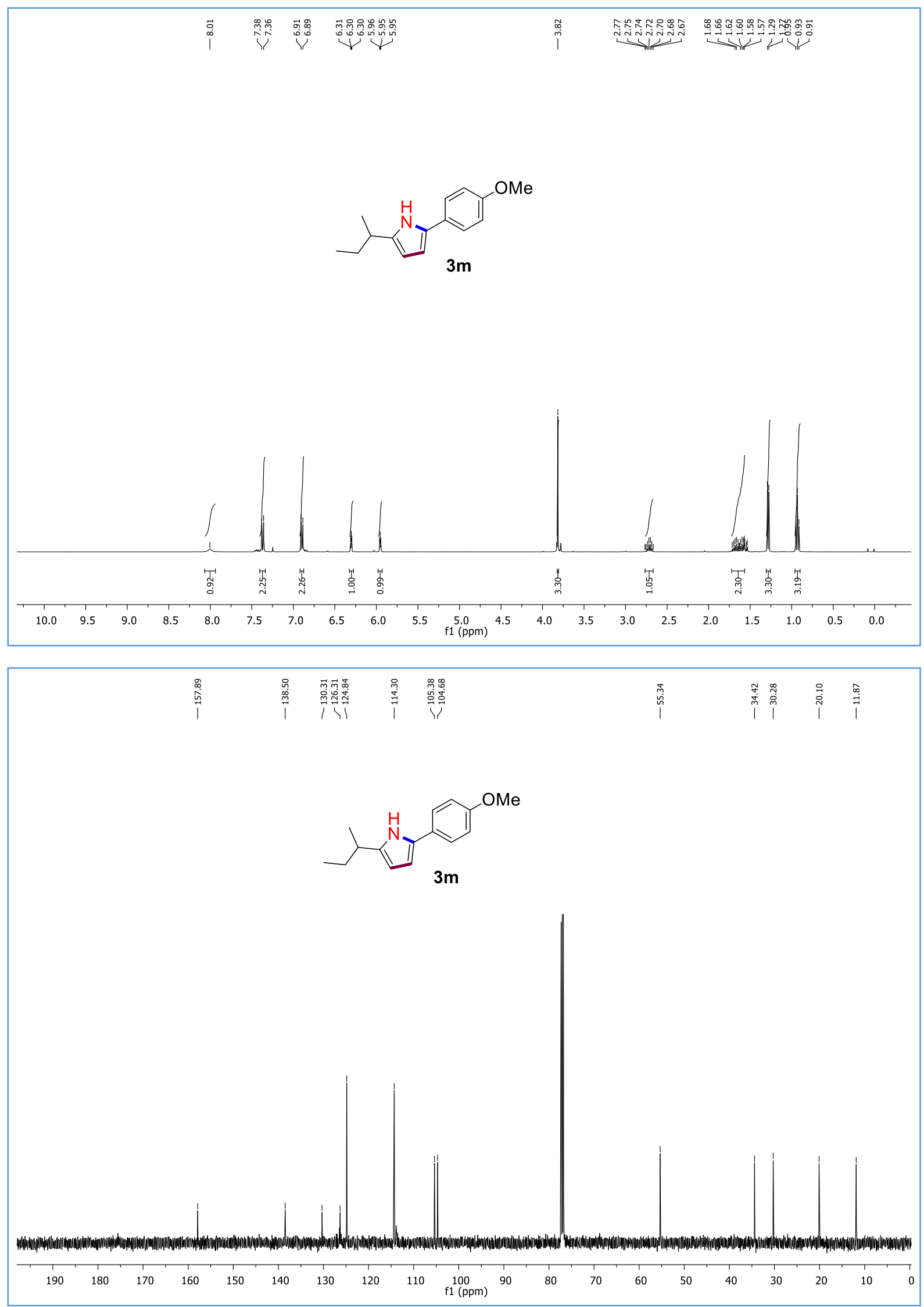

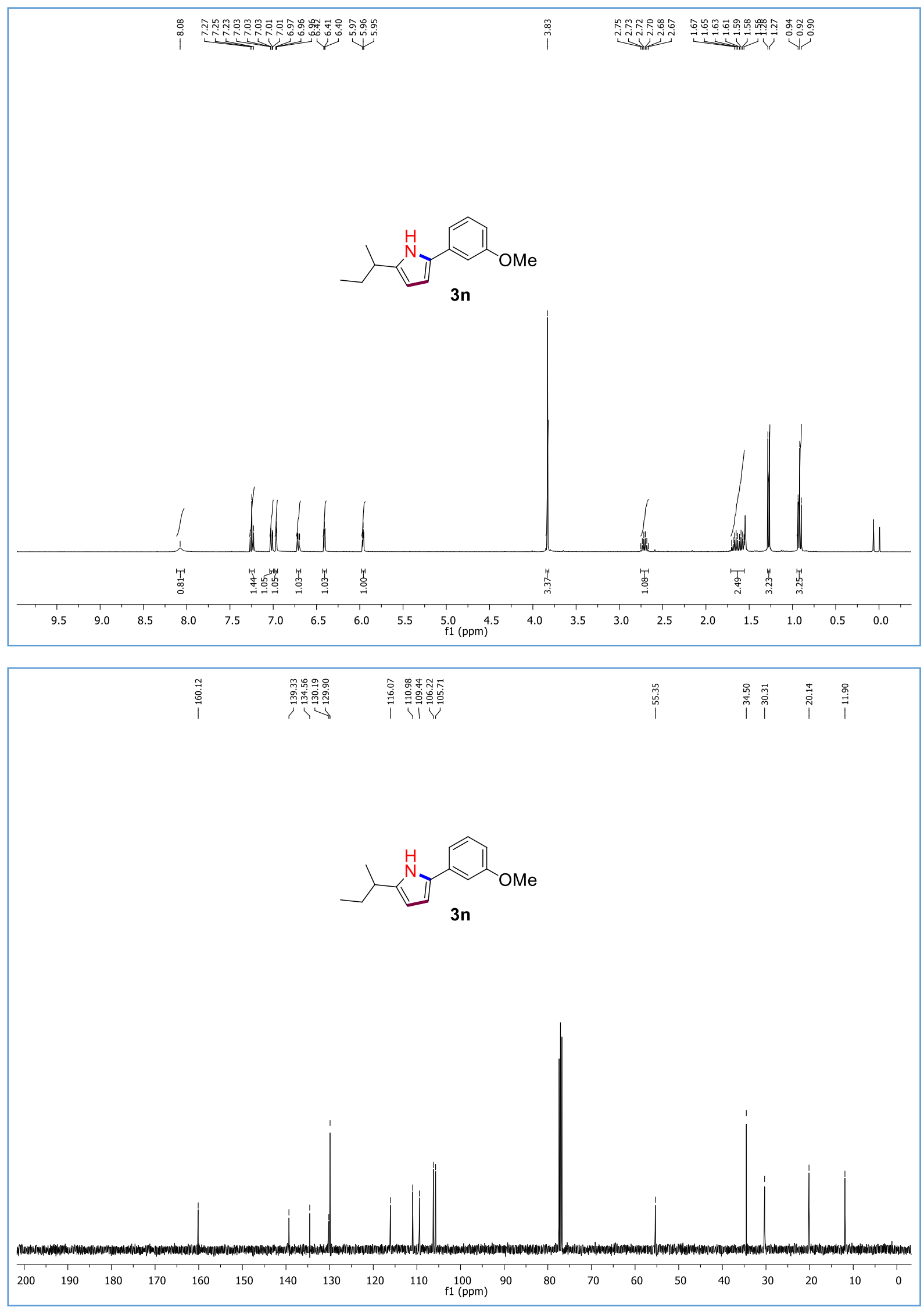

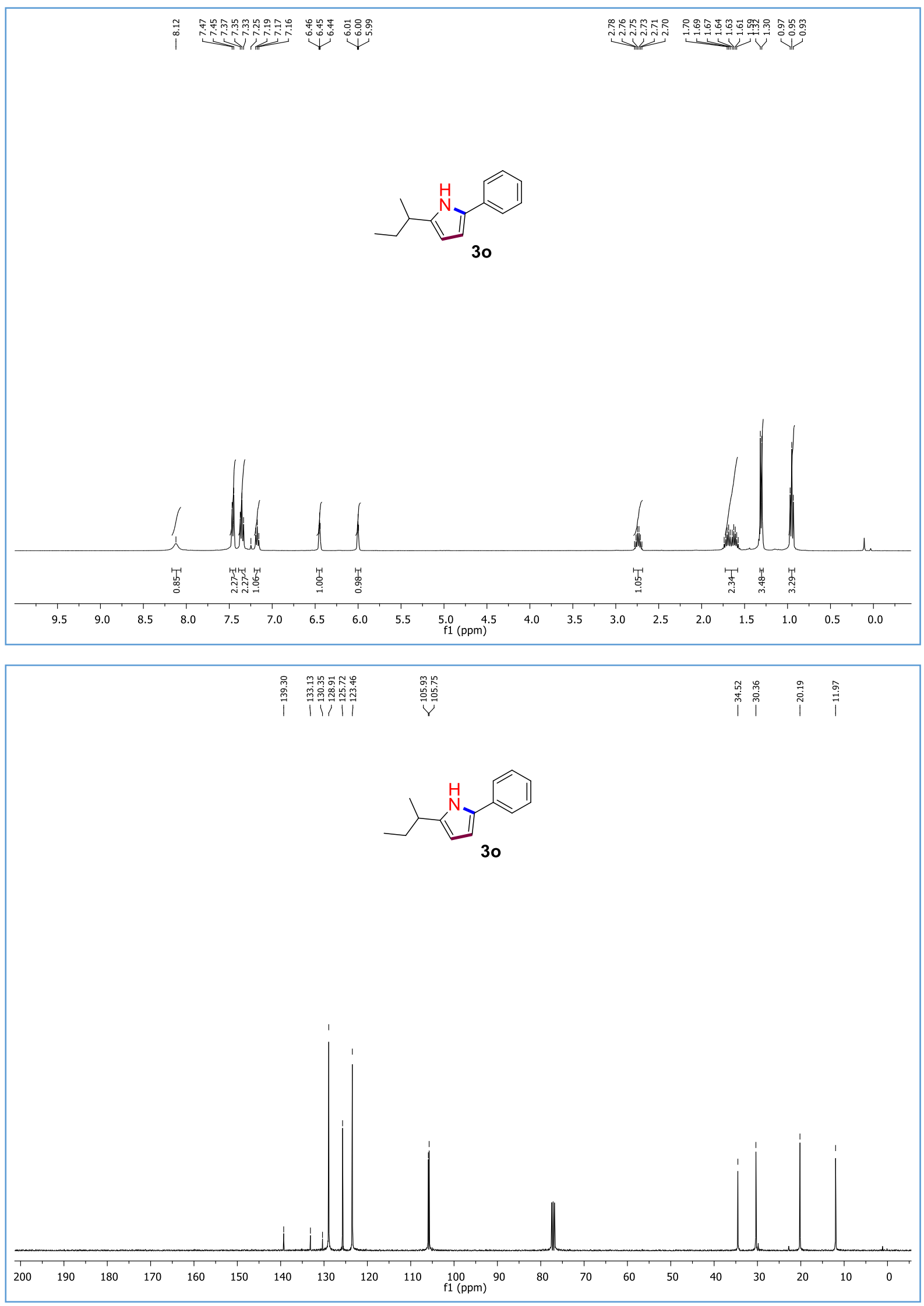

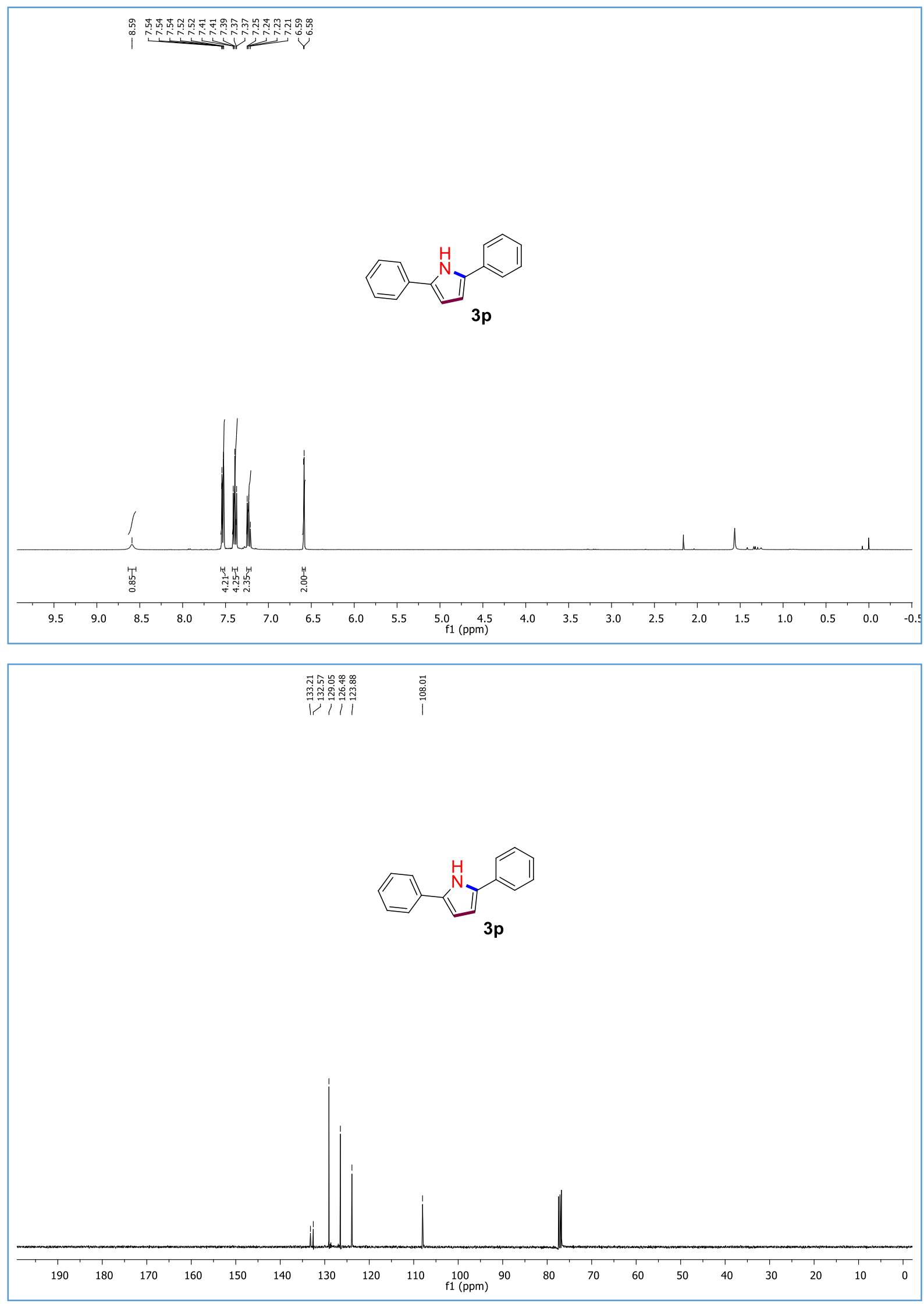

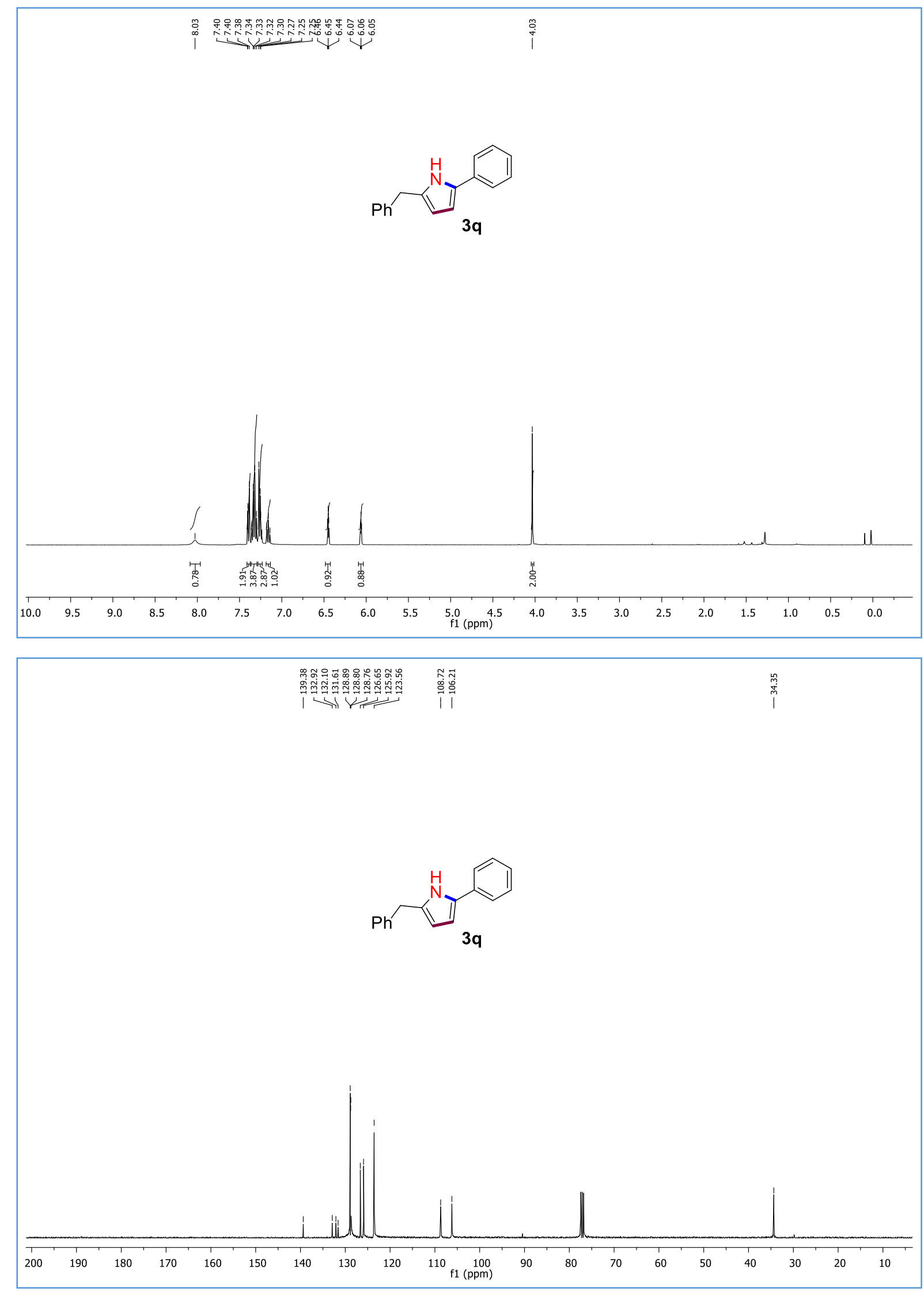

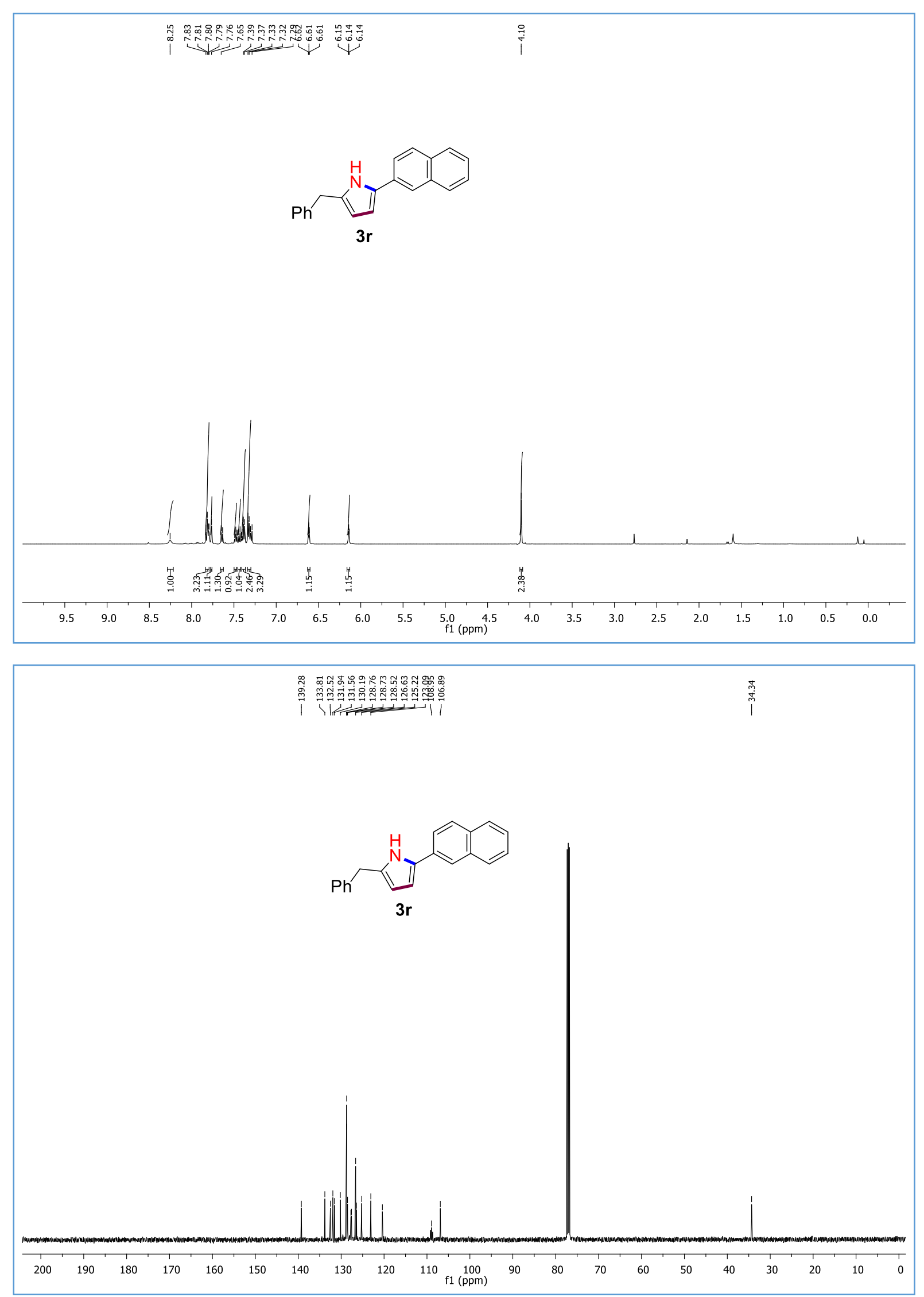

S27 

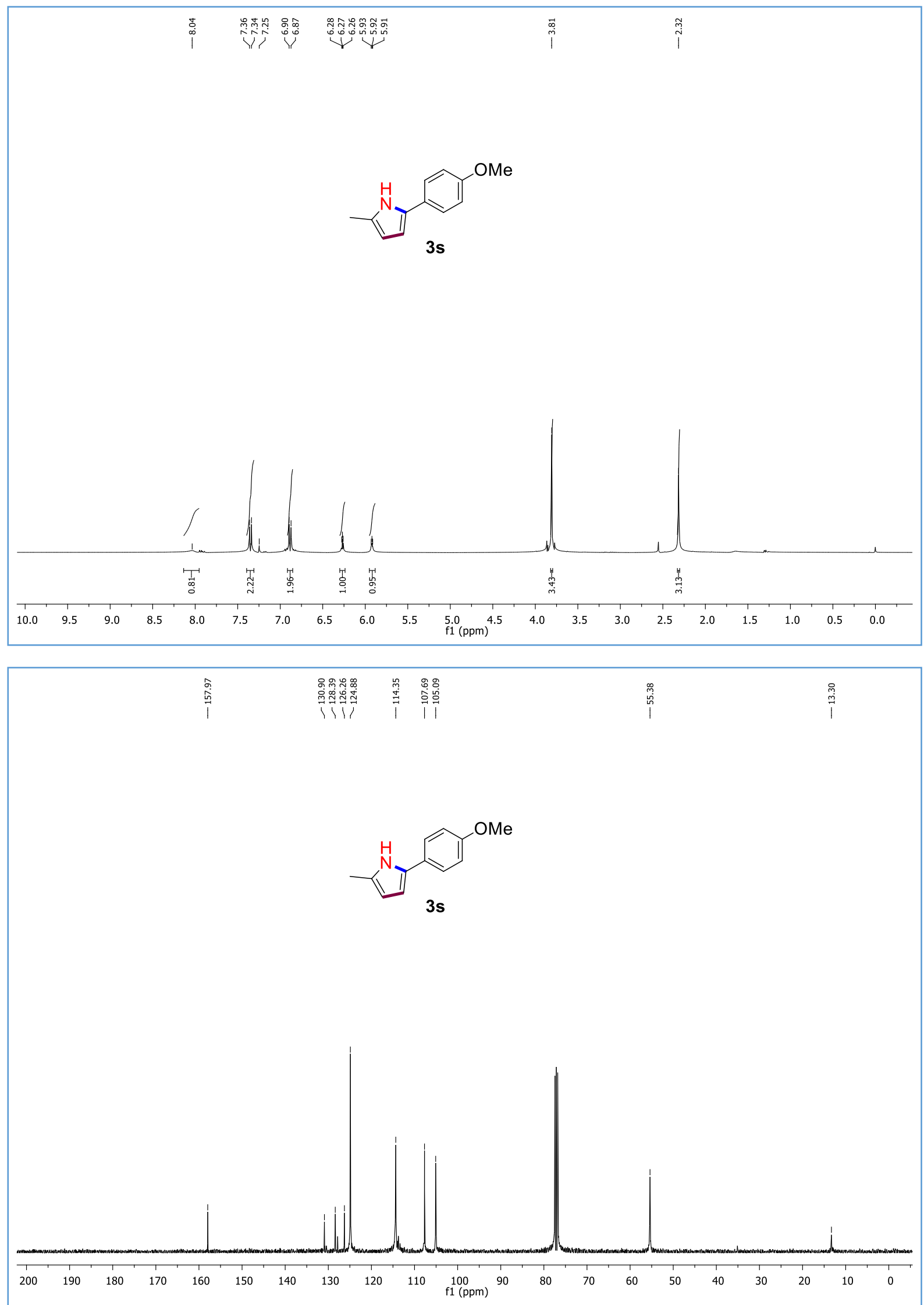

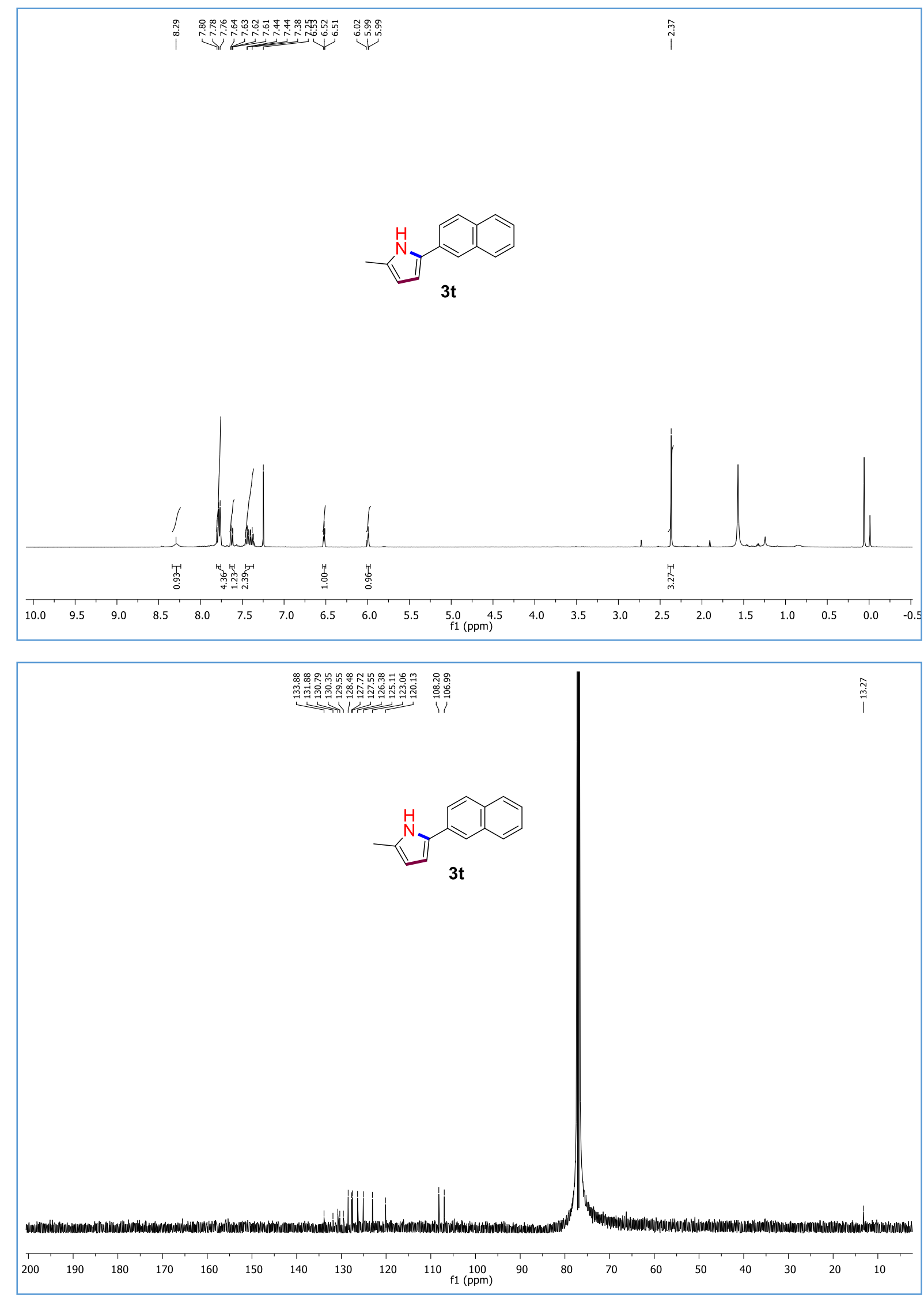

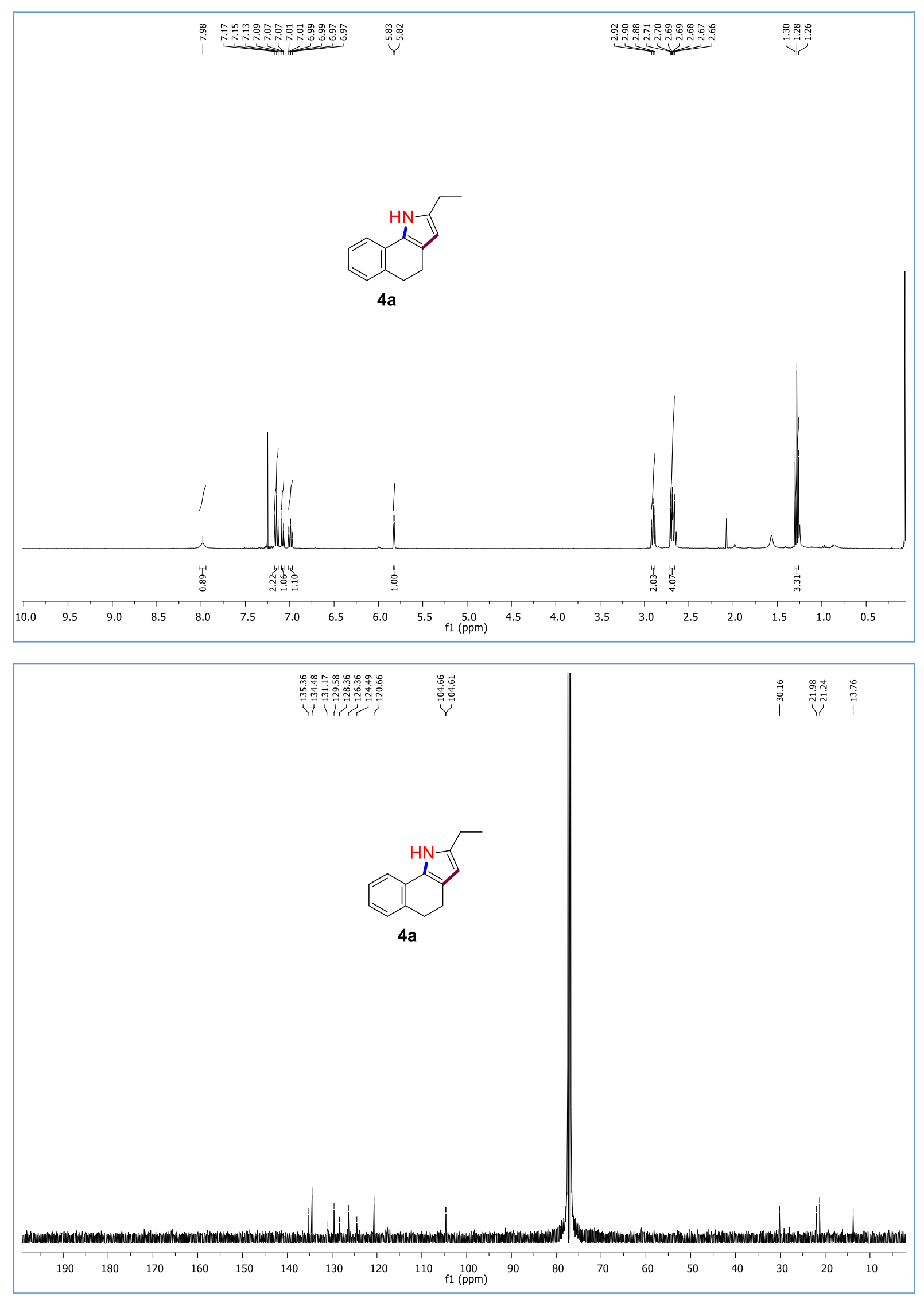

S30 

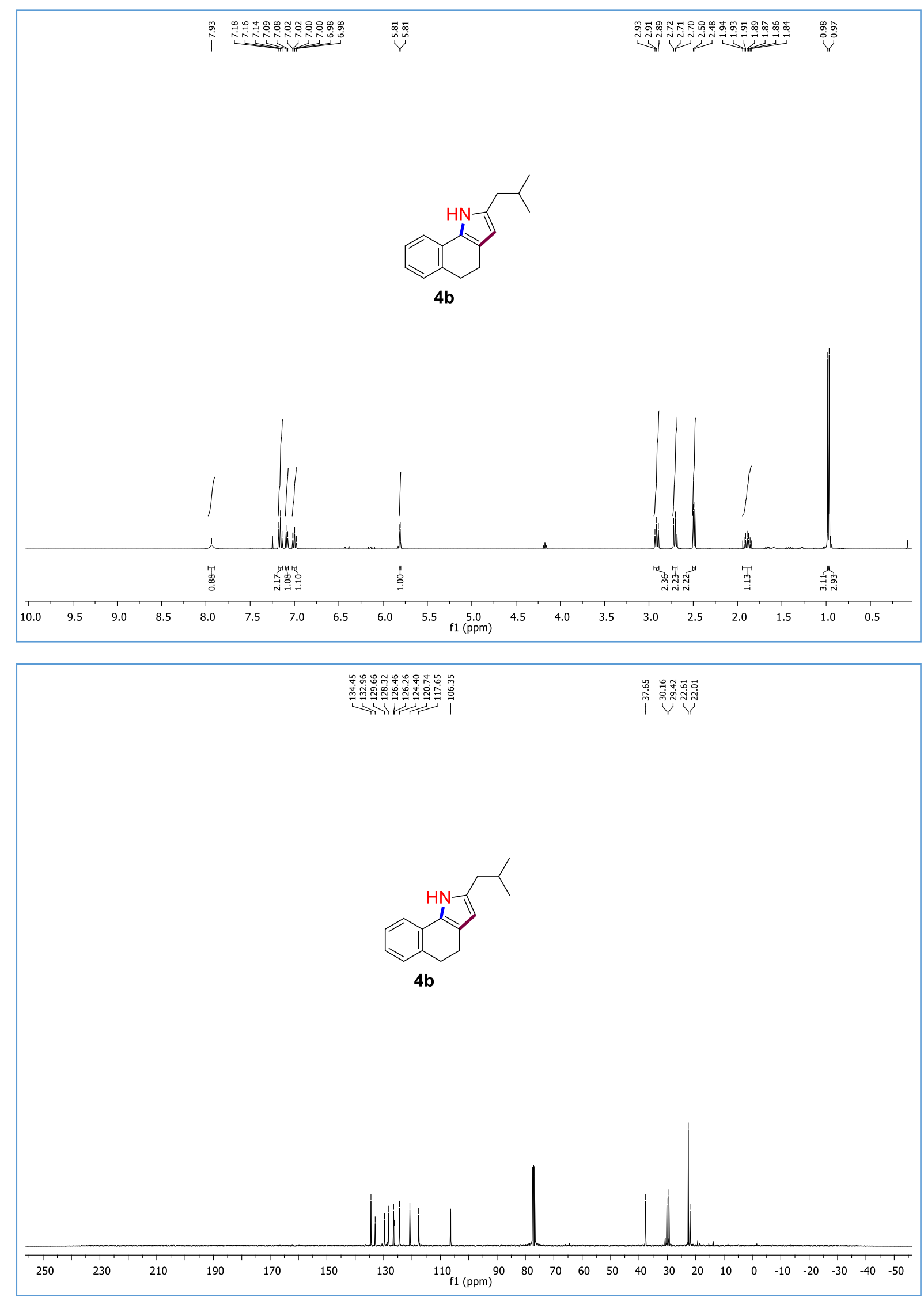

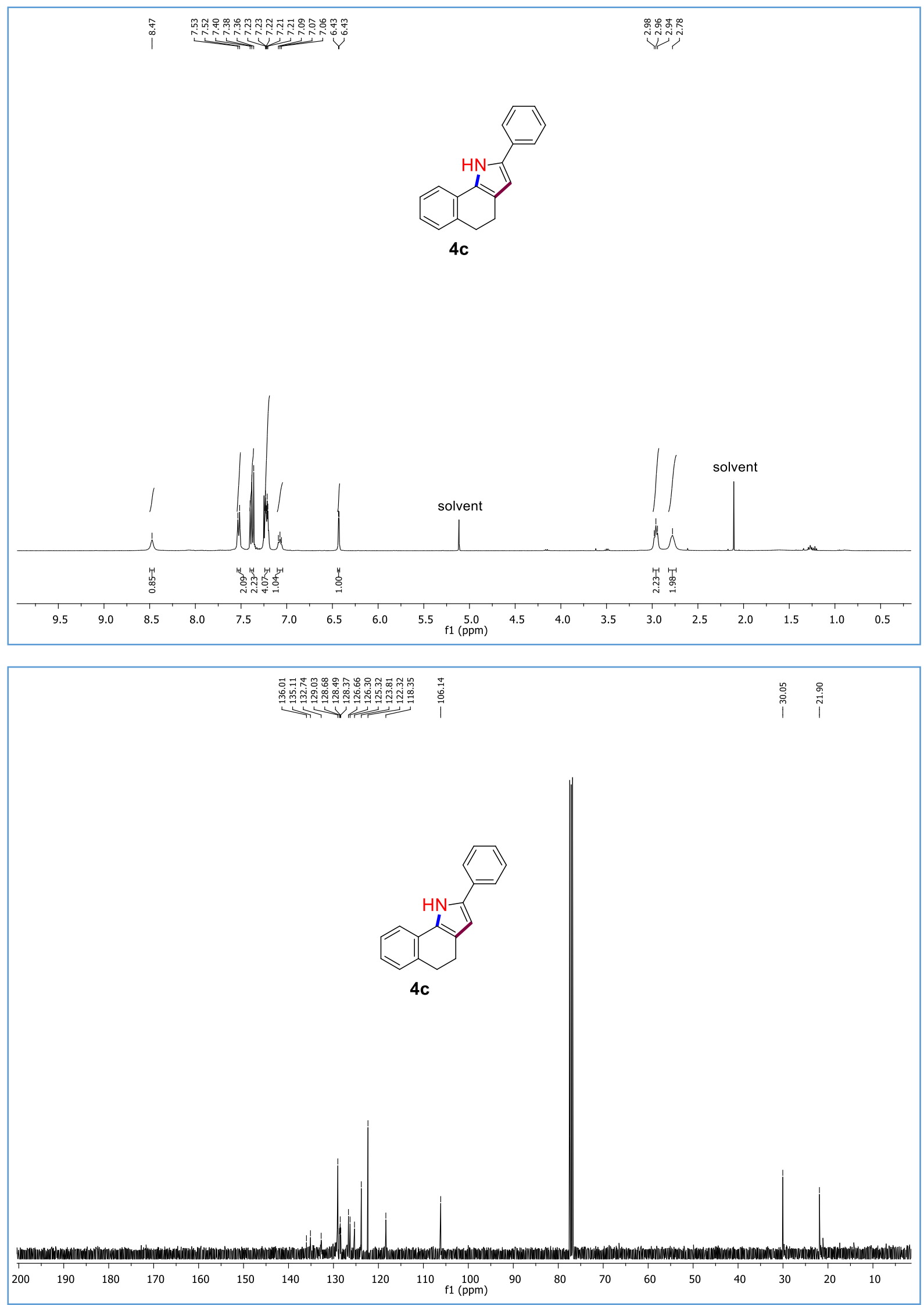

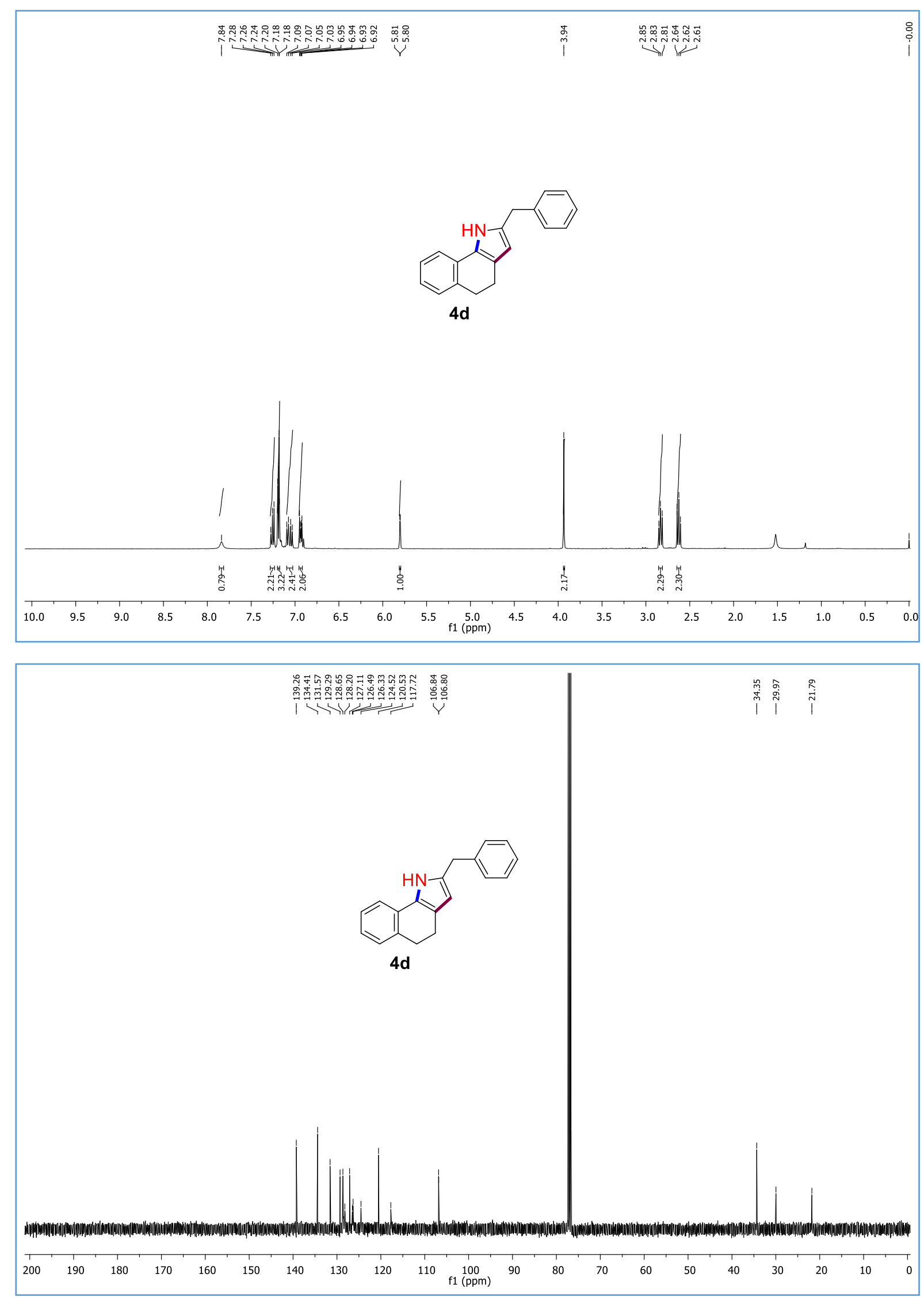

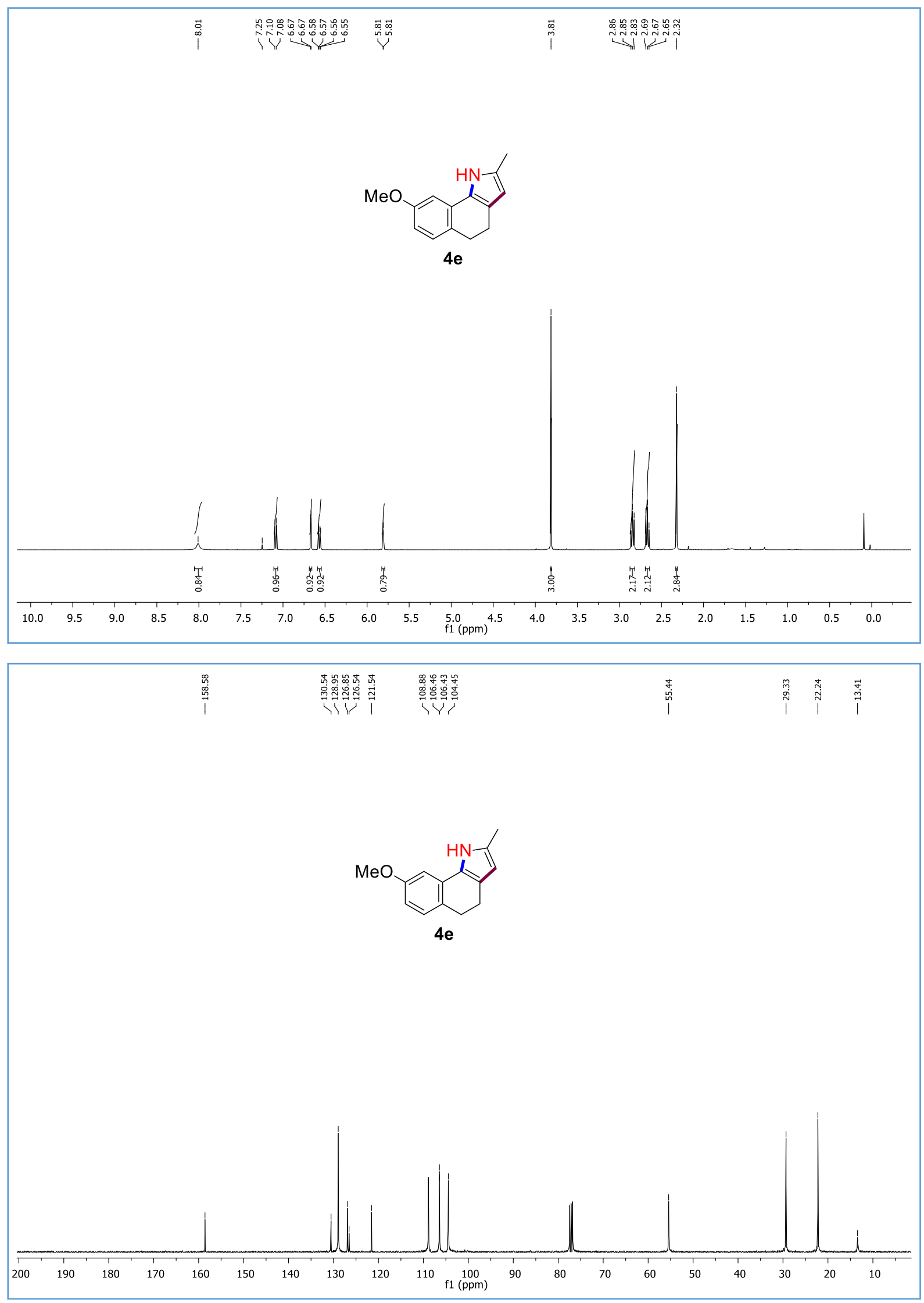

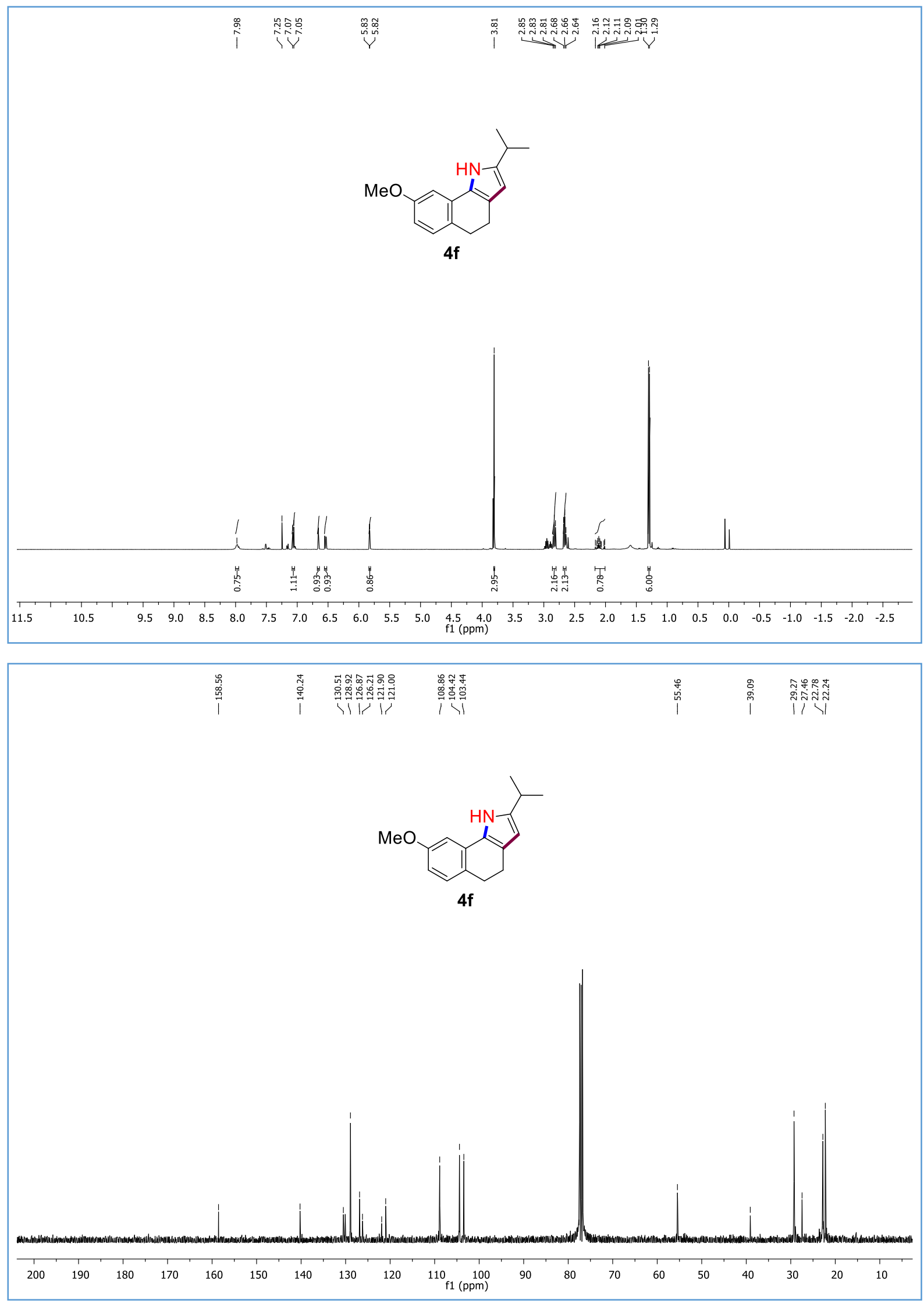

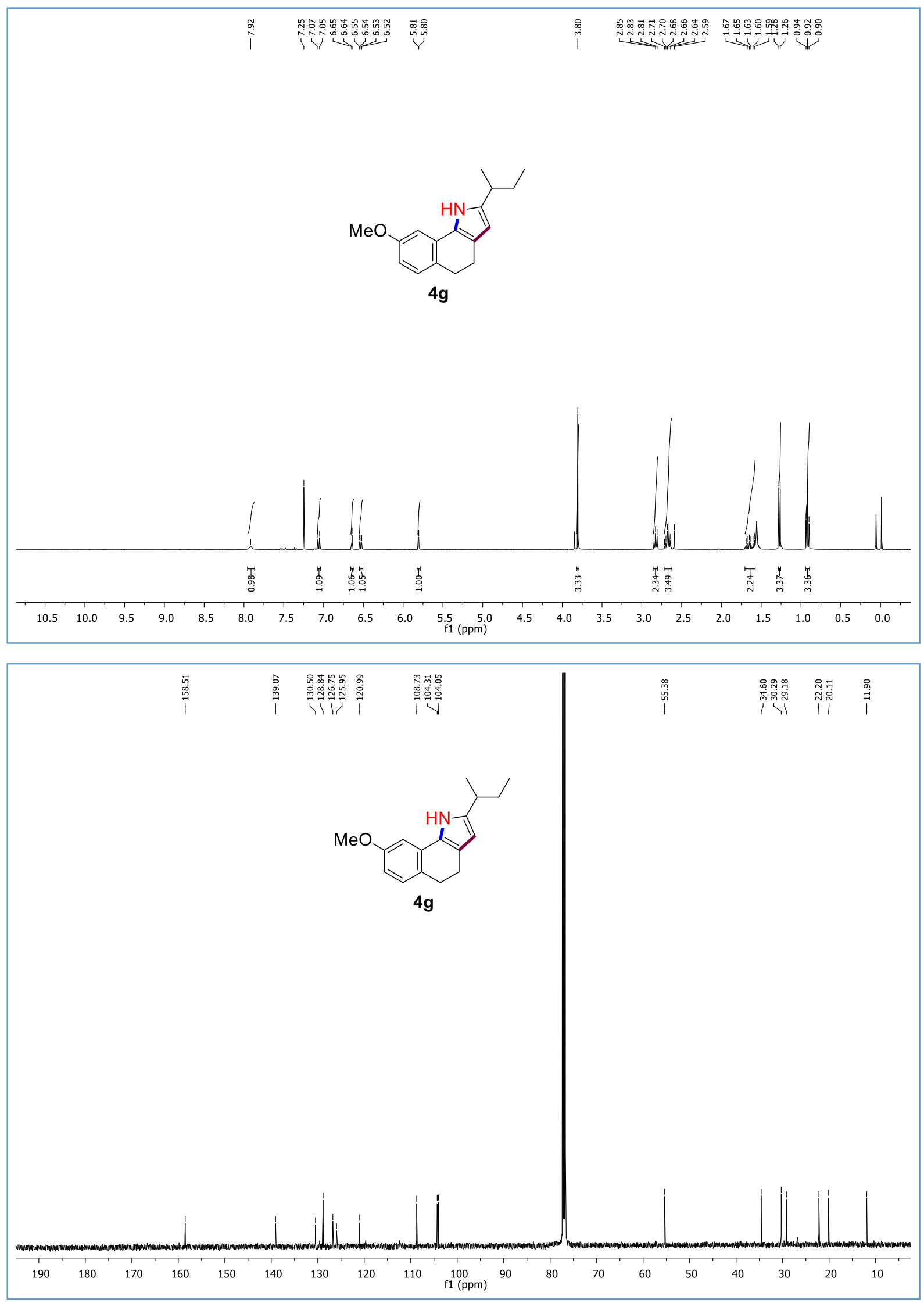

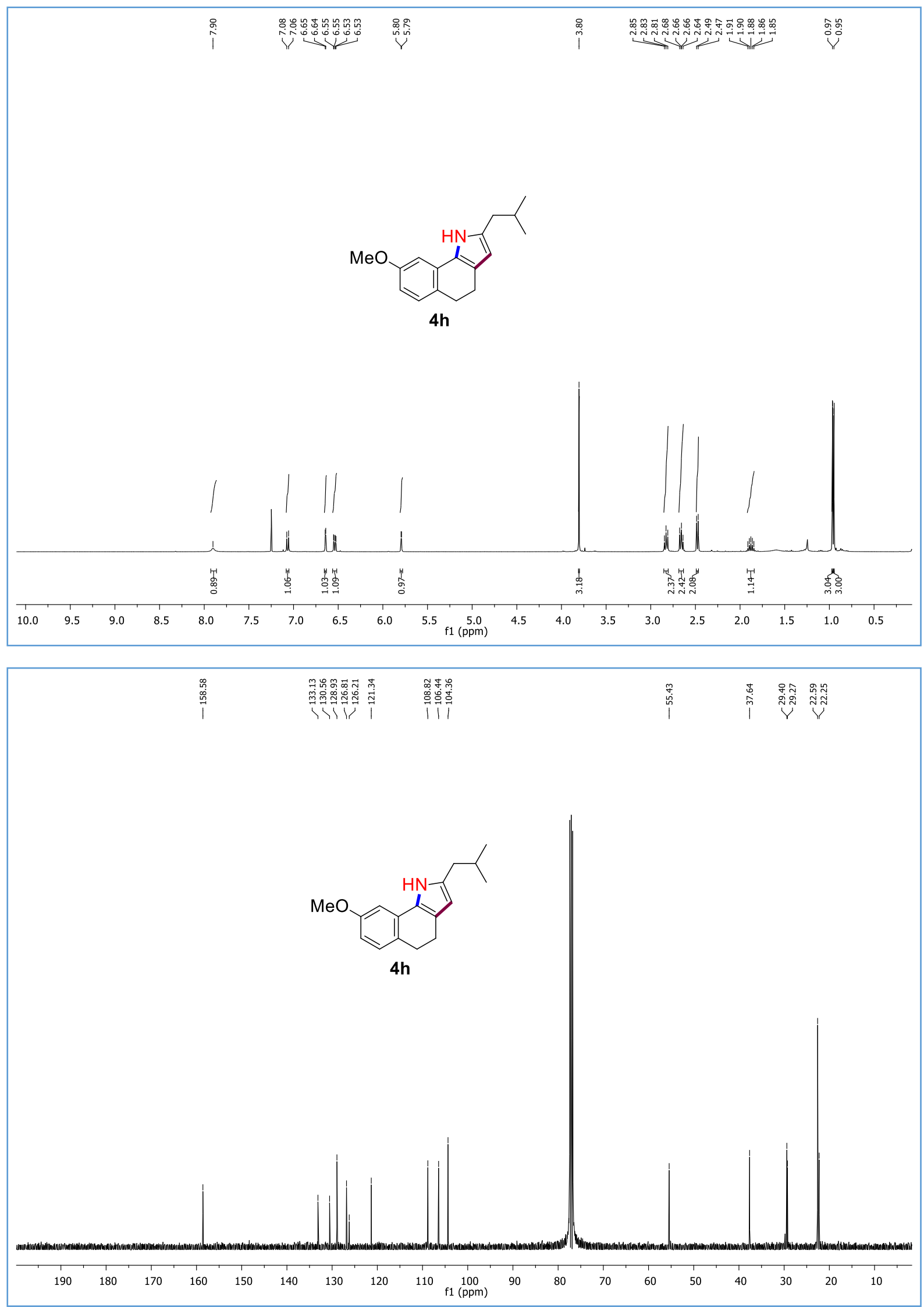

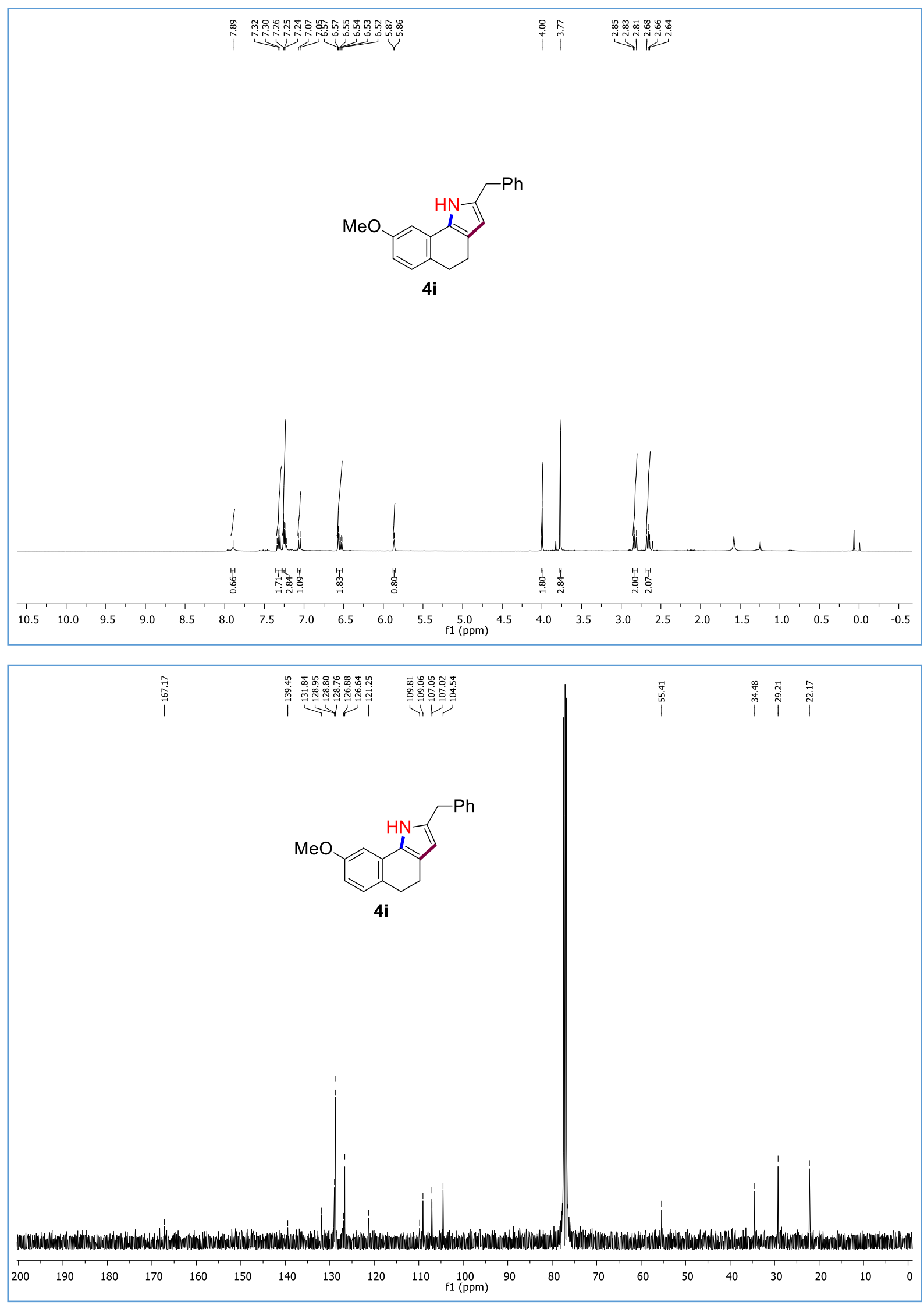

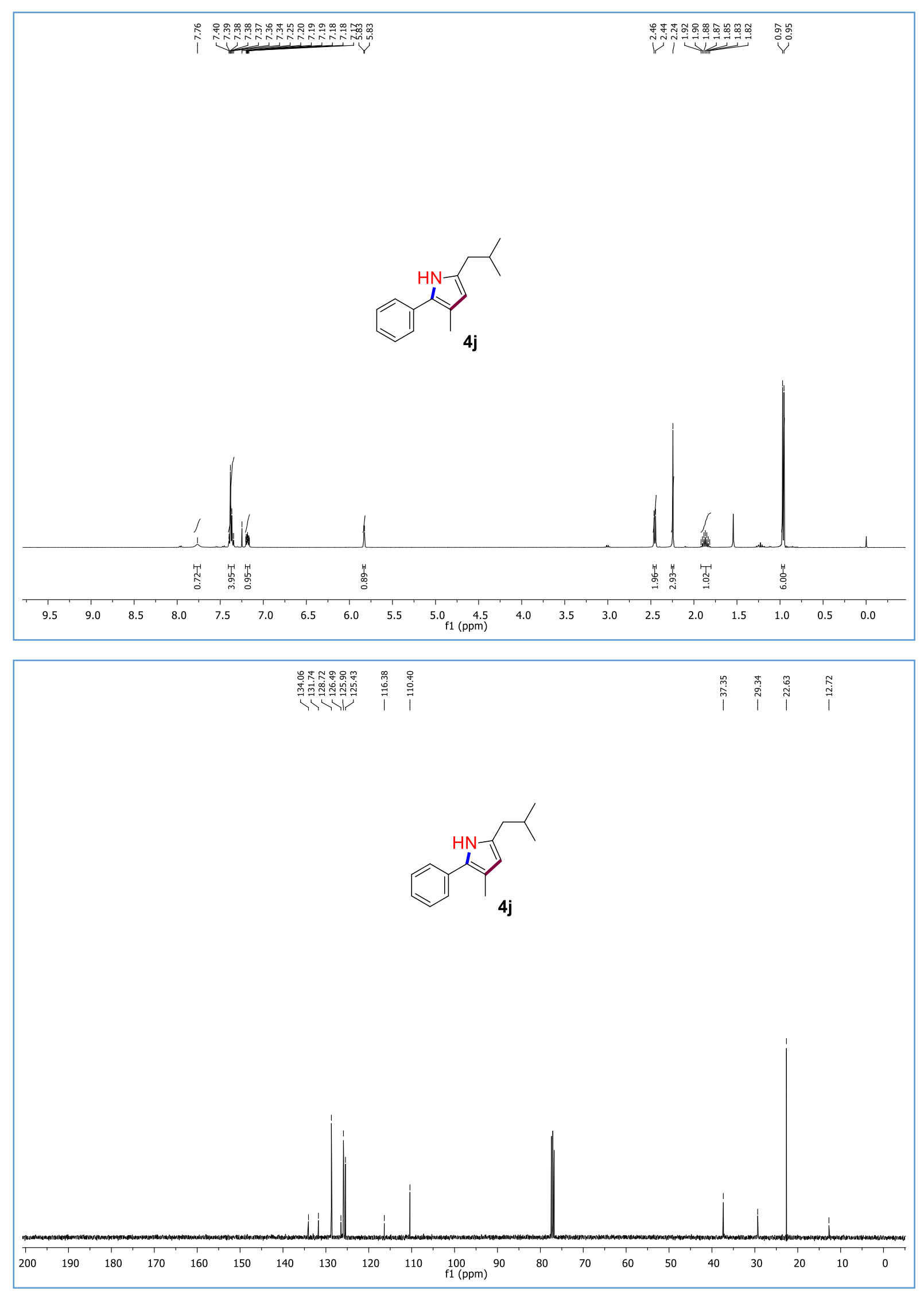

S39 


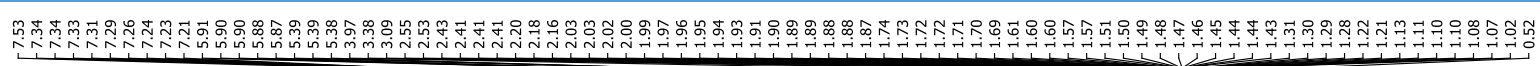
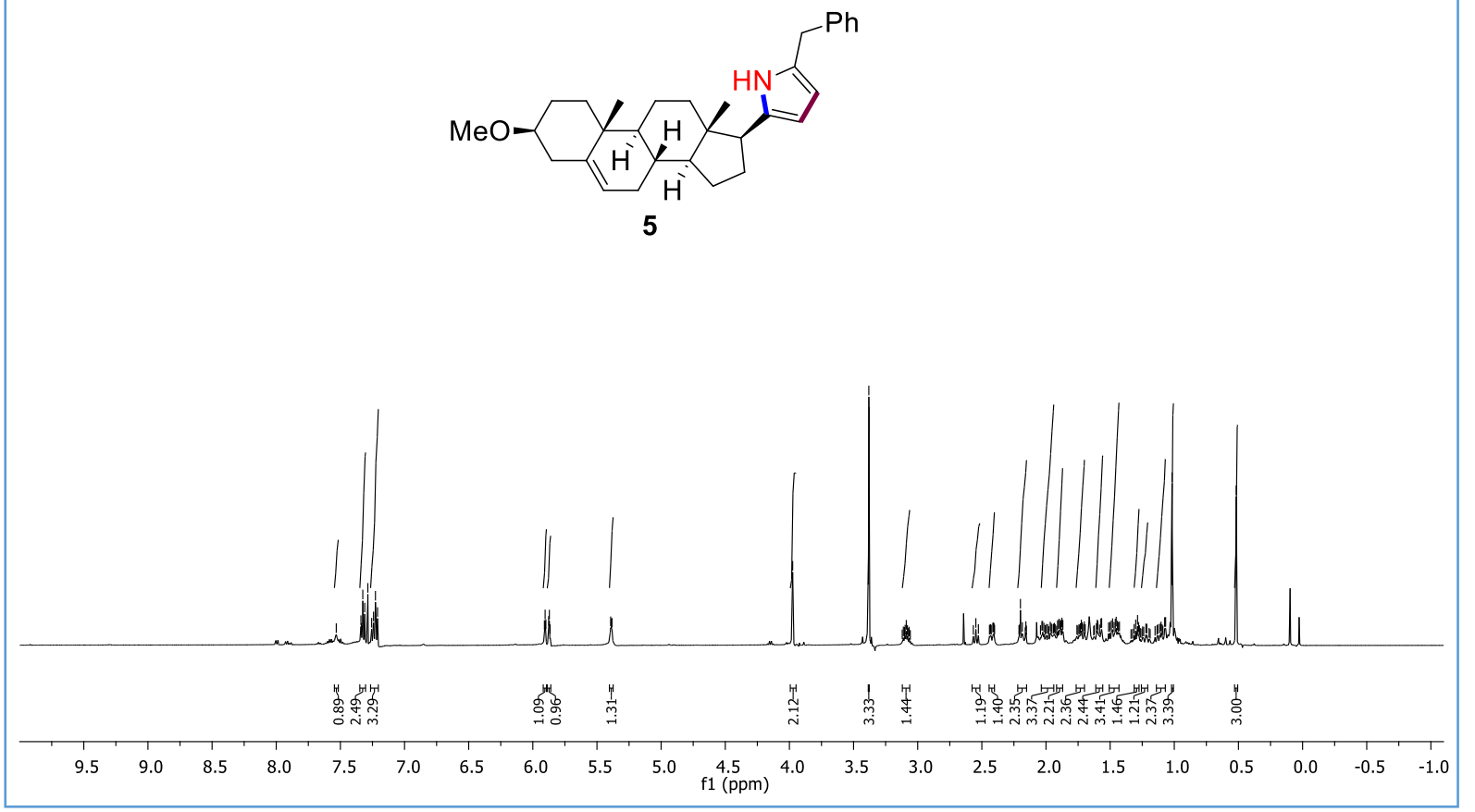

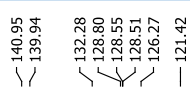

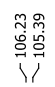

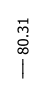

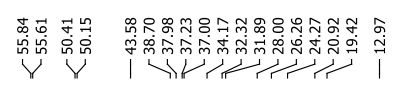

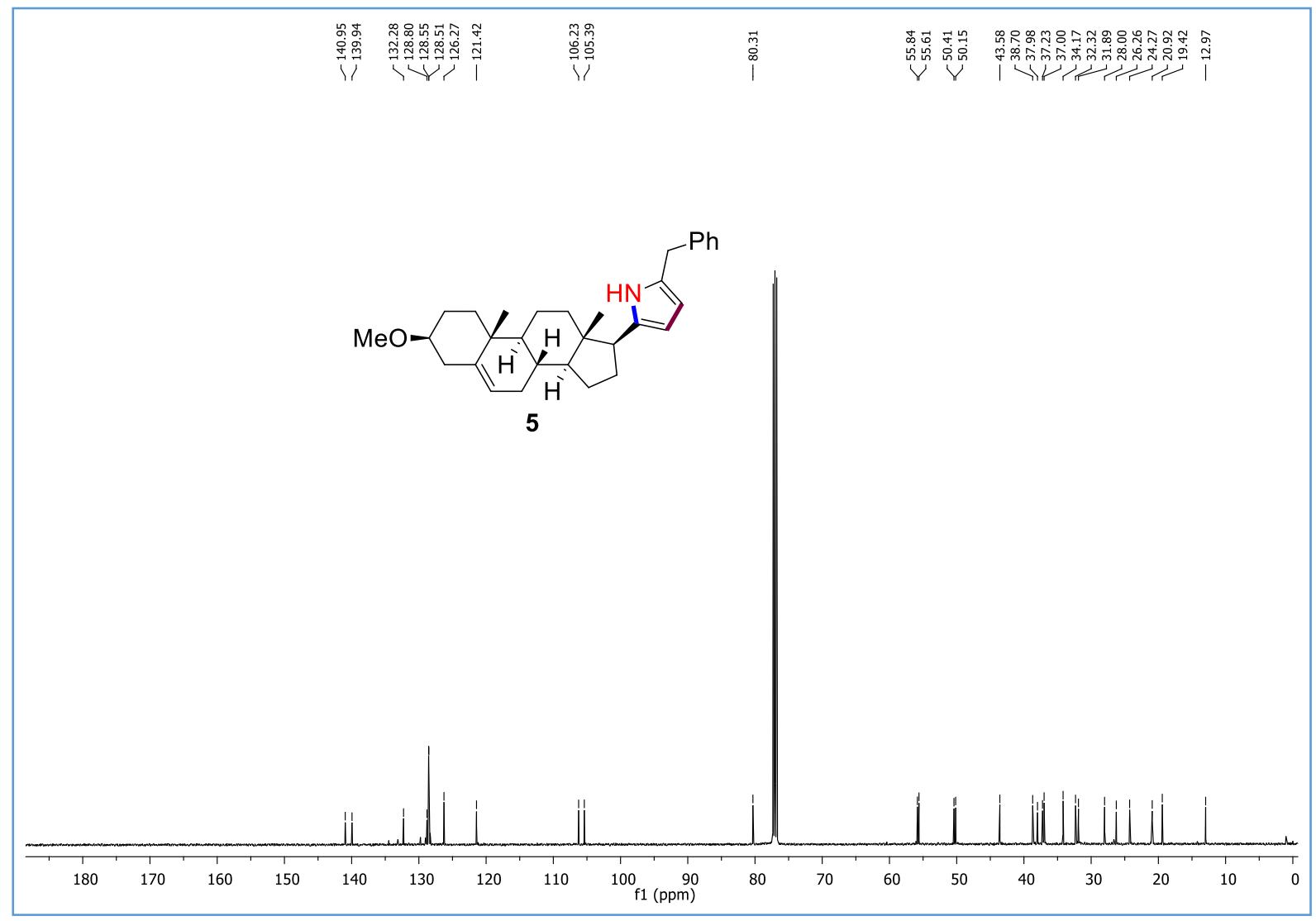

S40 

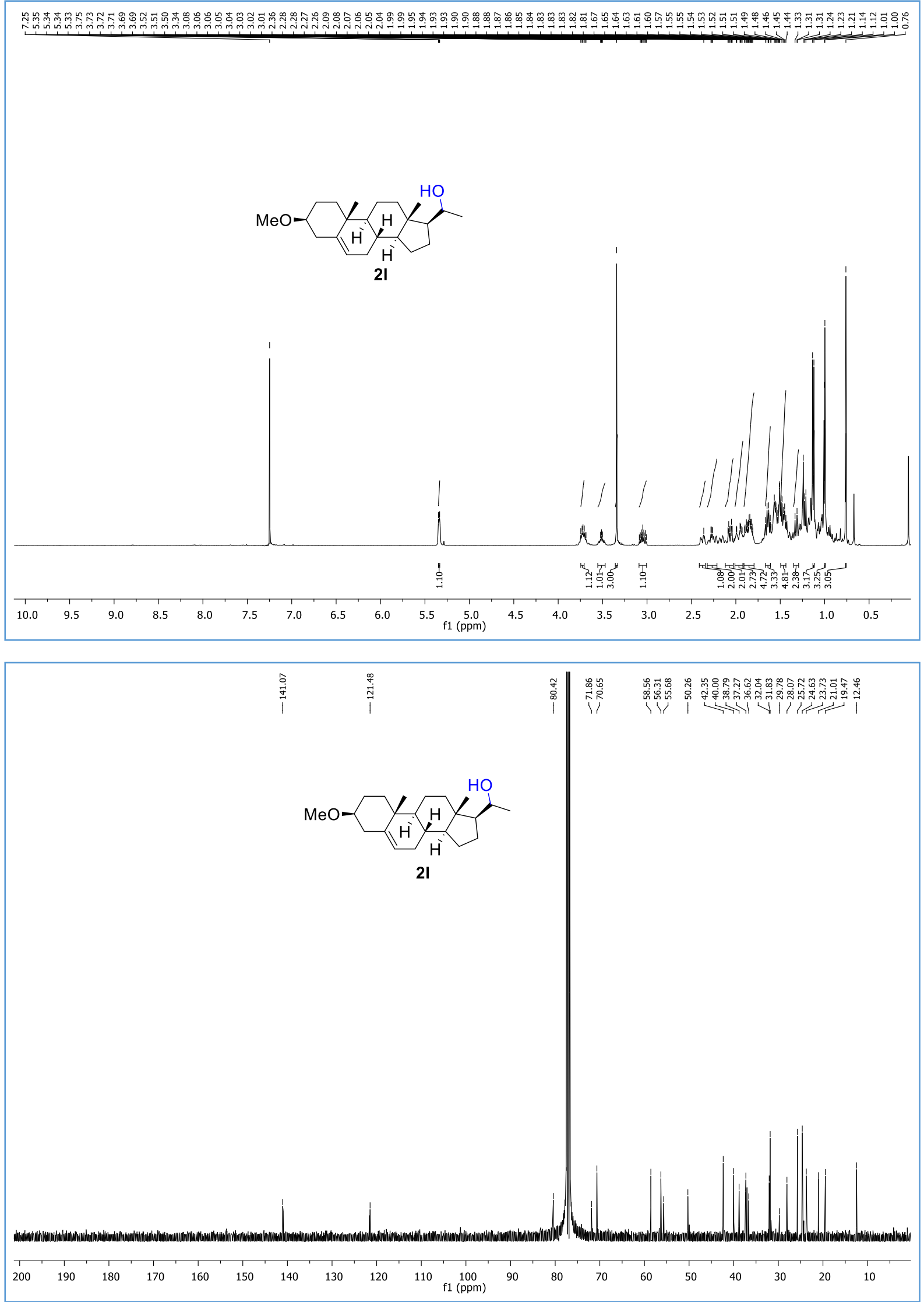